\title{
1 Breakup dynamics and dripping-to-jetting transition 2 in a Newtonian/shear-thinning multiphase 3 microsystem
}

4 Yong Ren*ab, Zhou Liu ${ }^{\text {ac }}$ and Ho Cheung Shum ${ }^{* a c}$

5 a Department of Mechanical Engineering, The University of Hong Kong, Pokfulam Road, $6 \quad$ Hong Kong.

7

${ }^{\mathrm{b}}$ Current Address: Department of Mechanical, Materials \& Manufacturing Engineering, The University of Nottingham, Ningbo, China.

${ }^{\mathrm{c}} H K U-S h e n z h e n$ Institute of Research and Innovation (HKU-SIRI), Shenzhen, Guangdong, China.

*Author to whom correspondence should be addressed.E-mail: yong.ren@nottingham.edu.cn; ashum@hku.hk; Tel: +852 28597904

The breakup dynamics in non-Newtonian multiphase microsystems are associated with a variety of industrial applications such as food production and biomedical engineering. In this study, we numerically and experimentally characterize the dripping-to-jetting transition under various flow conditions in a Newtonian/shear-thinning multiphase microsystem. Our work can help to predict the formation of undesirable satellite droplet, which is one of the challenges in dispensing nonNewtonian fluids. We also demonstrate the variations in breakup dynamics between shearthinning and Newtonian fluids under the same flow conditions. For shear-thinning fluids, droplet size increases when the Capillary number is smaller than a critical value, while decreases when Capillary number is beyond the critical value. The variations highlight the importance of rheological effects in flows with a non-Newtonian fluid. The viscosity of shear-thinning fluids significantly affects the control over droplet size, therefore necessitating the manipulation of the 
shear rate through adjusting the flow rate and the dimension of the nozzle. Consequently, the droplet size can be tuned in controlled manner. Our findings can guide the design of novel microdevices for generating droplets of shear-thinning fluids with predetermined droplet size.This enhances the ability to fabricate functional particles using an emulsion-templated approach. Moreover, elastic effects are also investigated experimentally using a model shearthinning fluid that also exhibits elastic behaviors: droplets are increasingly deformed with increasing elasticity of the continuous phase. The overall understanding in the model multiphase microsystem will facilitate the use of a droplet-based approach for non-Newtonian multiphase applications ranging from energy to biomedical sciences.

\section{Introduction}

Microfluidic multiphase system can be applied in a wide variety of applications. For example, it can facilitate drug encapsulation and release using emulsion droplets as a template to fabricate core-shell microspheres, capsules and other functional materials. ${ }^{1-3}$ The system can also be used for the generation of jets, which can work as precursors of microfibers for application in wound dressing and tissue engineering. ${ }^{2,-7}$ Nevertheless, the practical microfluidic multiphase applications increasingly demand the use of fluids with more complex rheological behaviors, such as non-Newtonian fluids, whose viscosities change with the applied stress. Non-Newtonian fluids are ubiquitous in daily life; examples include blood, lotions, creams, shampoos and toothpaste. They are also widely used in industrial applications such as inkjet printing, spraying and coating. ${ }^{8-9}$ Microscaled non-Newtonian multiphase systems have been increasingly applied in biomedical engineering, food production, and energy applications. For instance, an electrokinetic microdevice has been developed to convert fluidic mechanical energy to electrical energy by 
46 means of electrokinetic phenomena such as streaming currents. ${ }^{9}$ By reducing the hydrodynamic

47 conductance while maintaining the same streaming current, addition of an appropriate polymer

48 to the working fluid in a microchannel can enhance the energy conversion efficiency, which is a

49 ratio of electrical output power and hydrodynamic input power. ${ }^{10-11}$

50 Non-Newtonian multiphase microsystem has become a subject of intense research, and it is of

51 paramount importance to understand the relevant physical phenomena, one of which involves the

52 deformation of liquid threads and subsequently droplet formation. The performance of the

53 droplets is intimately tied to the ability to control droplet size, which has a strong impact on the

54 droplet stability as well as optical and mechanical properties. ${ }^{12}$ For example, when the droplets

55 are used as a template to fabricate micro/nano particles for the drug delivery system, the shape

56 and size of emulsion droplets have significant impacts on the drug release kinetics. ${ }^{13-}$

$57{ }^{14}$ Monodisperse droplets with precisely controlled sizes can be used to deliver an accurate dosing

58 of contained payload such as drug, flavoring, or chemical reactants. ${ }^{15}$ Therefore, monodispersity

59 and size tenability are highly desired for ensuring that the droplets exhibit constant, controlled

60 and predictable behaviors. ${ }^{4,12}$ However, the complex rheological properties of non-Newtonian

61 fluids challenge the versatility in droplet size control. For example, the stretching and/or thinning

62 of non-Newtonian liquid filaments will lead to the formation of "bead-on-string" patterns. ${ }^{16-}$

$63{ }^{17}$ These beads can subsequently become undesirable satellite droplets, increasing the

64 polydispersity of the resultant droplet population. The dynamics of the droplet formation process

65 can be characterized into a dripping and a jetting regime. The dripping-to-jetting transition can

66 be estimated using the Capillary number of the continuous phase, $C a_{\text {out }}$, a ratio of viscous force to

67 surface tension, and the Weber number of the dispersed phase, $W e_{i n}$, a ratio of inertial force to

68 surface tension. ${ }^{18}$ 


$$
W e_{i n}=\frac{\rho_{D P} d v_{D P}^{2}}{\sigma}
$$

$$
C a_{\text {out }}=\frac{\eta_{C P, 0} v_{C P}}{\sigma}
$$

71 where $\rho_{D P}$ is the density of dispersed phase, $d$ refers to the diameter of the orifice, $V_{D P}$ and $V_{C P}$

72 are the velocities of dispersed and continuous phase respectively, $\sigma$ is the interfacial tension,

73 and $\eta_{C P, 0}$ is the apparent viscosity of the non-Newtonian fluid of continuous phase. The subscript

74 " 0 " refers to zero-shear rate when a shear-dependent fluid is used. Droplet formation occurs at

75 the orifice directly after the two fluids meetin the dripping process, when both $C a_{\text {out }}$ and $W e_{\text {in }}$ are

76 small, as surface tension dominates. By contrast, jetting occurs when $C a_{\text {out }}$ or $W e_{\text {in }}$ is large, as the

77 viscous stress or the inertial force on the droplet will be large enough to overcome surface tension. Droplets are generated after breakup of a jet at some distance downstream in this 79 regime. While the dynamics of droplet breakup has been systematically investigated in 80 Newtonian fluids, ${ }^{17-18}$ the validity of the understanding has not been adequately confirmed in 81 non-Newtonian fluid systems. In addition, a number of non-Newtonianfluids such as polymeric 82 solutions, whole blood orprotein solutions with large polymeric molecules often exhibit elastic 83 property due to the stretching and coiling of the polymer chains. The resultant non-Newtonian 84 rheological behaviors inspire interesting applications. ${ }^{19}$ For instance, microparticles driven in 85 viscoelastic solutions can migrate toward the centerline of a microchannel because of the first 86 normal stress difference between the centerline and the walls; three-dimensional (3D) particle 87 focusing can be achieved via a synergistic combination of inertial andelastic forces. ${ }^{20}$ The 88 elasticity of the focusing fluid has been shown to facilitate formation of smaller droplets. ${ }^{21}$ These 
89

90

91

92

examples attests to the need for a comprehensive understanding of the role of elastic fluids with shear-rate-dependent viscosity in droplet formation using microfluidic systems.

In this paper, we focus on a shear-thinning fluid, which is one of the most frequently encountered non-Newtonian fluids, ${ }^{22}$ and investigate the dripping-to-jetting transition of a Newtonian/shear-thinning two-phase system and characterize the formation of satellite drops in the model system. The breakup time and droplet size are compared with Newtonian/Newtonian two-phase system at the same Weber number and Capillary number.We demonstrate that the degree of control over droplet size can be enhanced by adjusting the flow rate and nozzle size, thus tuning the shear rate experienced by the non-Newtonian fluids. We also present an experimental study of the elasticity effect of a shear-thinning fluid on droplet generation in a microfluidic two-phase system. The shape of the droplets are shown to vary with the Weissenberg number, which represents a ratio of the relaxation time to the hydrodynamic time. Our work helps to elucidate the effects of rheological behaviors on the breakup dynamics in a Newtonian/non-Newtonian two-phase microscaled flow; our understanding inspires new approaches to control sizes and shapes of functional droplets in applications requiring the use of non-Newtonian fluids.

\section{Numerical model}

The microcapillary co-flow device has been widely used in emulsion generation. ${ }^{23} \mathrm{~A} 3 \mathrm{D}$ numerical model with the same design is established in the present investigation (see Fig.1a). A Newtonian fluid is injected in a cylindrical capillary as the dispersed phase at a constant mean velocity $V_{D P}$. This inner fluid is surrounded by a non-Newtonian outer phase, which is injected through the coaxial square capillary as the continuous phase at a constant mean velocity $V_{C P}$. The 
111

112

113

114

115

116

117

118

119

120

121

122

123

124

125

126

127

128

129

130

cross section (perpendicular to the main flow direction) consists of a circle inside a square (see inset of Fig.1a). Thus, the continuous phase is flowing through a cross section between an inner circle and outer square. No-slip condition is applied at the solid boundaries of the walls of capillaries. Gauge pressure of zero is applied at the outlet of the domain. The maximum entrance length is $9.4 \mu \mathrm{m}$ in our numerical computations, and the length of the computational domain is 6 $\mathrm{mm}$, which is long enough to ensure that the fluid flow can be fully developed. The governing equations for incompressible two-phase fluids are, ${ }^{24}$

$$
\begin{gathered}
\frac{\partial \alpha_{C P}}{\partial t}+v \cdot \nabla \alpha_{C P}=0 \\
\frac{\partial \rho}{\partial t}+\nabla \cdot(\rho v)=0 \\
\frac{\partial}{\partial t}(\rho v)+\nabla \cdot(\rho v v)=-\nabla P+\nabla \cdot\left[\eta\left(\nabla v+\nabla v^{T}\right)\right]+\frac{\rho \sigma \nabla \alpha_{C P}}{\frac{1}{2}\left(\rho_{D P}+\rho_{C P}\right)} \nabla \cdot \frac{\nabla \alpha_{C P}}{\left|\nabla \alpha_{C P}\right|} \\
\rho=\alpha_{C P} \rho_{C P}+\left(1-\alpha_{C P}\right) \rho_{D P} \\
\eta=\alpha_{C P} \eta_{C P}+\left(1-\alpha_{C P}\right) \eta_{D P}
\end{gathered}
$$

where $v$ is the flow velocity, $P$ is the pressure, $\rho$ is the volume averaged density, $\alpha_{C P}$ and $\rho_{C P}$ are the volume fraction and density of the continuous phase respectively, $\eta_{D P}$ and $\eta_{C P}$ are dynamic viscosities of the dispersed and continuous phases, respectively. The 3D numerical volume of fluid (VOF) model has been implemented to investigate the droplet breakup in pair of nonNewtonian/Newtonian fluids in T-shaped microchannel. ${ }^{24}$ The rheological property of the nonNewtonian continuous phase is modeled using Cross model, which has been widely applied in the modeling of industrial shear-thinning fluids. ${ }^{25} \mathrm{~A}$ shear-thinning fluid is used in our present study and its apparent viscosity is defined by, 


$$
\eta_{C P}=\eta_{\infty}+\frac{\eta_{0}-\eta_{\infty}}{1+\left(\lambda_{c} \dot{\gamma}\right)^{n}}
$$

132

133

where $\eta_{\infty}$ refers to lower limiting values of the fluid viscosity or infinite shear viscosity; $\eta_{0}$ refers to upper limiting values of the fluid viscosity or zero shear viscosity; $\lambda_{c}$ is the time constant, the reciprocal of which corresponds to a critical shear rate that provides a useful indicator of the onset shear rate for shear thinning; $\dot{\gamma}$ is the shear rate and $n$ represents the power law index of fluid. The solutions with different values of these parameters will have different rheological behaviors. Any effect due to gravity is neglected for simplicity, because the length scale of interest is much smaller than the capillary lengthof $1.33 \mathrm{~mm}$ in our model. The two-phase flow is solved using the VOF method by CFD software Ansys Fluent 14.0. The approach has been successfully demonstrated to investigate multiphase flow in microsystem. ${ }^{26}$ The governing equations are discretized to algebraic equations by using a control-volume-based technique. An iterative solver was deployed to solve the control-volume discretized equations. The iterative timestep is $10^{-7} \mathrm{~s}$ and the solution converges when the residual is below a tolerance set as 1.0 $\mathrm{x} 10^{-6}$.The simulations were performed using numerical grids composed of triangular elements, as shown in Fig.1b.The numerical data was subsequently analyzed by Ansys CFX-Post 14.0 after simulation was completed. Computational sensitivity study has been carried out to evaluate the effect of different triangular grid sizes. The generated morphology of interface as indicated by the green color between dispersed and continuous phases is compared using different grid sizes. Similar morphology is found at flow rates of the dispersed phase $Q_{D P}=50 \mathrm{ml} / \mathrm{h}$, and of the continuous phase $Q_{C P}=70 \mathrm{ml} / \mathrm{h}$, as shown in Fig.2. The size of interface gradually increases along the axial direction, and subsequently adopts cylindrical shape downstream with a uniform size for grid sizes of $4 \mu \mathrm{m}$ and $8 \mu \mathrm{m}$.The variation in the generated size of interface is less than $5 \%$ after convergence between grid sizes of $4 \mu \mathrm{m}$ and $8 \mu \mathrm{m}$. Furthermore, we investigated the grid 
154 size effect by comparing numerical results of axial velocity profile along transversal direction $(x$ 155 direction) for different grid sizes, at two downstream locations, $z=0.02 \mathrm{~mm}$, where the two 156 phases meet in vicinity of orifice, and $\mathrm{z}=5.27 \mathrm{~mm}$, where the flow has become fully developed, 157 as shown in Fig.3a-b, respectively. At $z=0.02 \mathrm{~mm}$, the sharp peak in the middle represents the 158 velocity profile of dispersed phase, which is purged out of orifice with very small size, thus the 159 velocity will be increased dramatically due to mass conservation. The magnitude of velocity for 160 the continuous phase is much smaller, because of the much larger cross section. At $z=5.27 \mathrm{~mm}$, 161 the velocity profiles of dispersed and continuous phases adjoin with a smooth transition at the 162 interface. Compared with coarse grids with a size of $12 \mu \mathrm{m}$, a good agreement is found between 163 refined grids with a size of $4 \mu \mathrm{m}$ and $8 \mu \mathrm{m}$ respectively. 4300956 triangular elements with a size 164 of $8 \mu \mathrm{m}$ are used in all the following numerical simulations to reduce computation cost. 


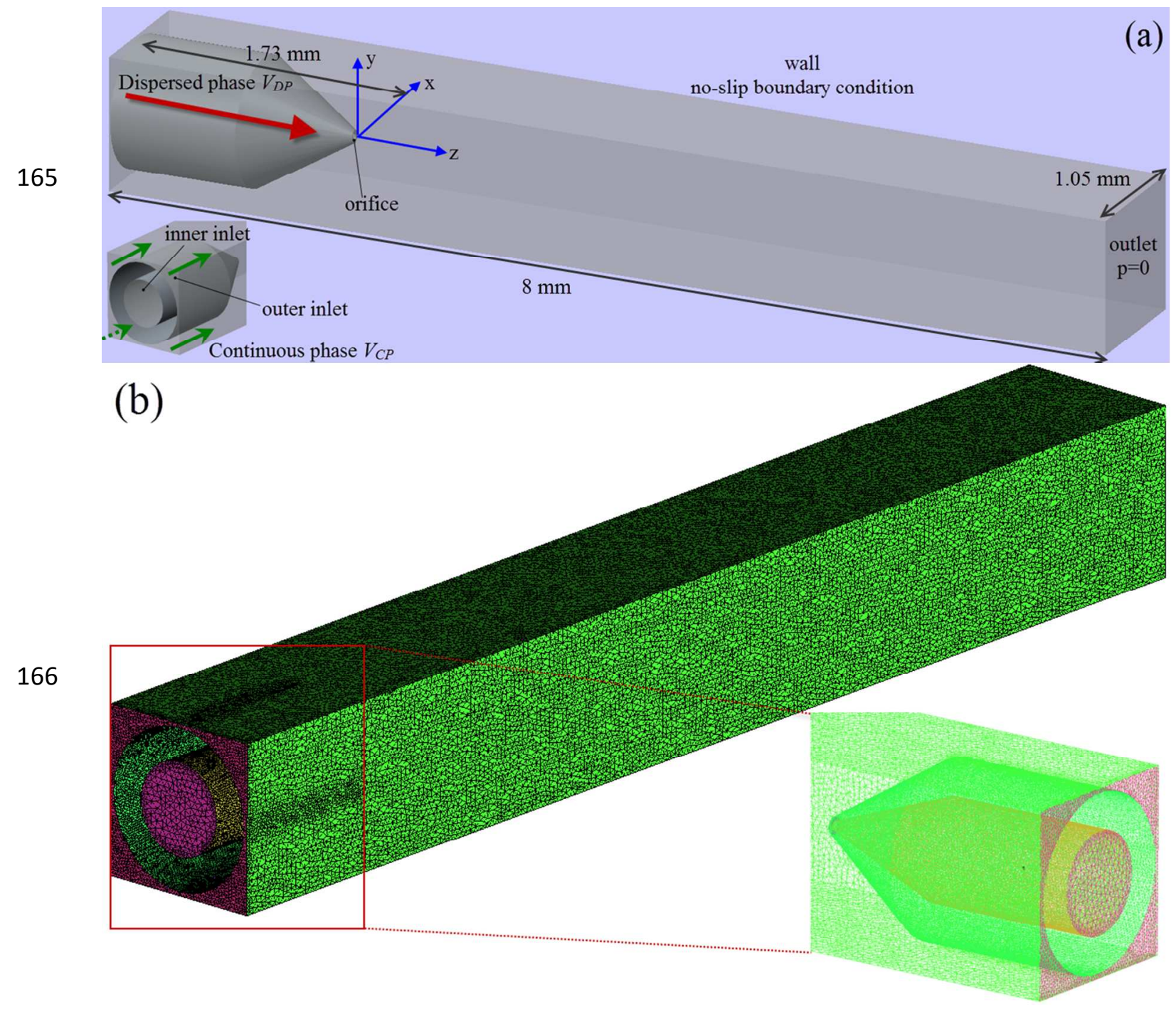

167 Fig.1(a) Schematic of the computational domain of multiphase microfluidic system. (b) Meshing grids. The close-up view of meshing of nozzle and inlets is shown in inset. 


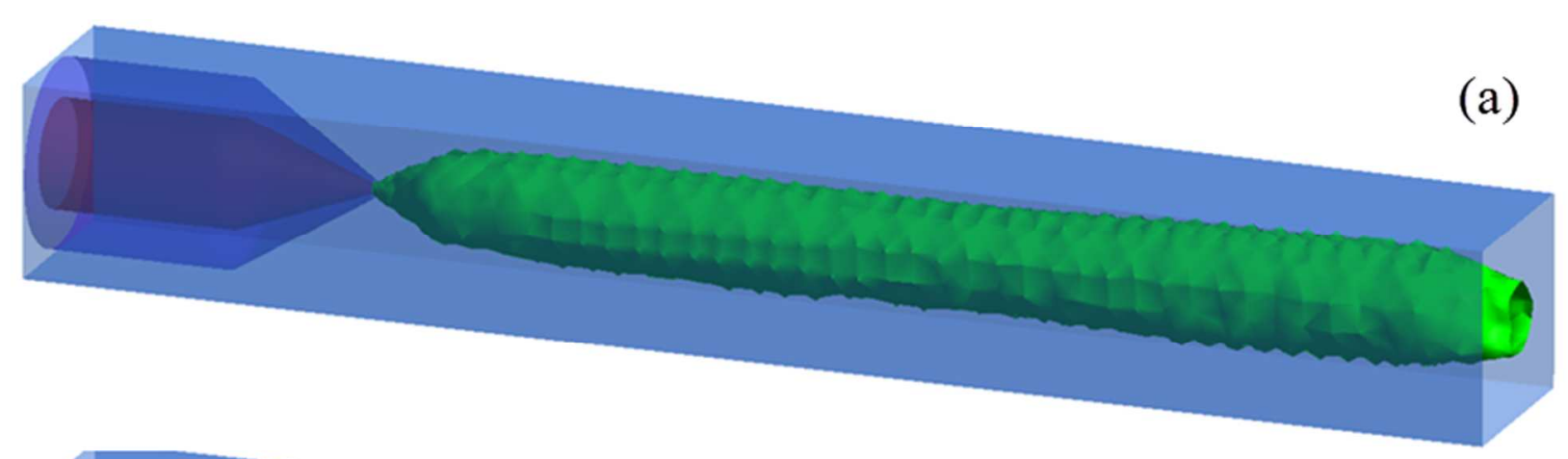

(b)

171

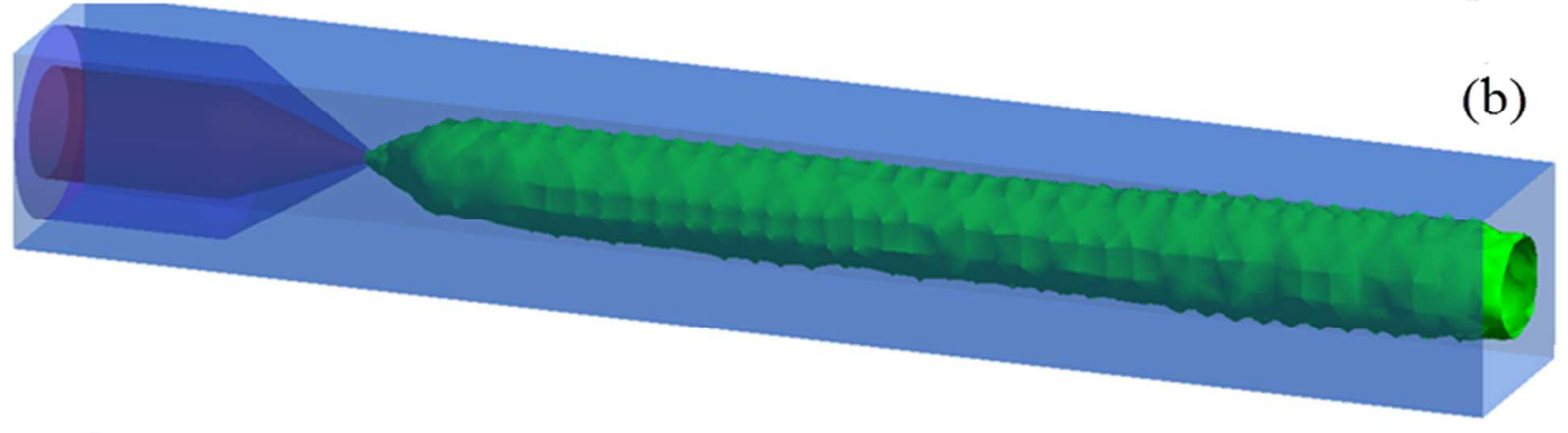

(c)

172 Fig.2 Comparison of morphology of jet using different meshing grid sizes (a) $0.012 \mathrm{~mm}$ (b) $1730.008 \mathrm{~mm}$ and (c) $0.004 \mathrm{~mm} \cdot Q_{D P}=50 \mathrm{ml} / \mathrm{h}, Q_{C P}=70 \mathrm{ml} / \mathrm{h}$. 
(a)

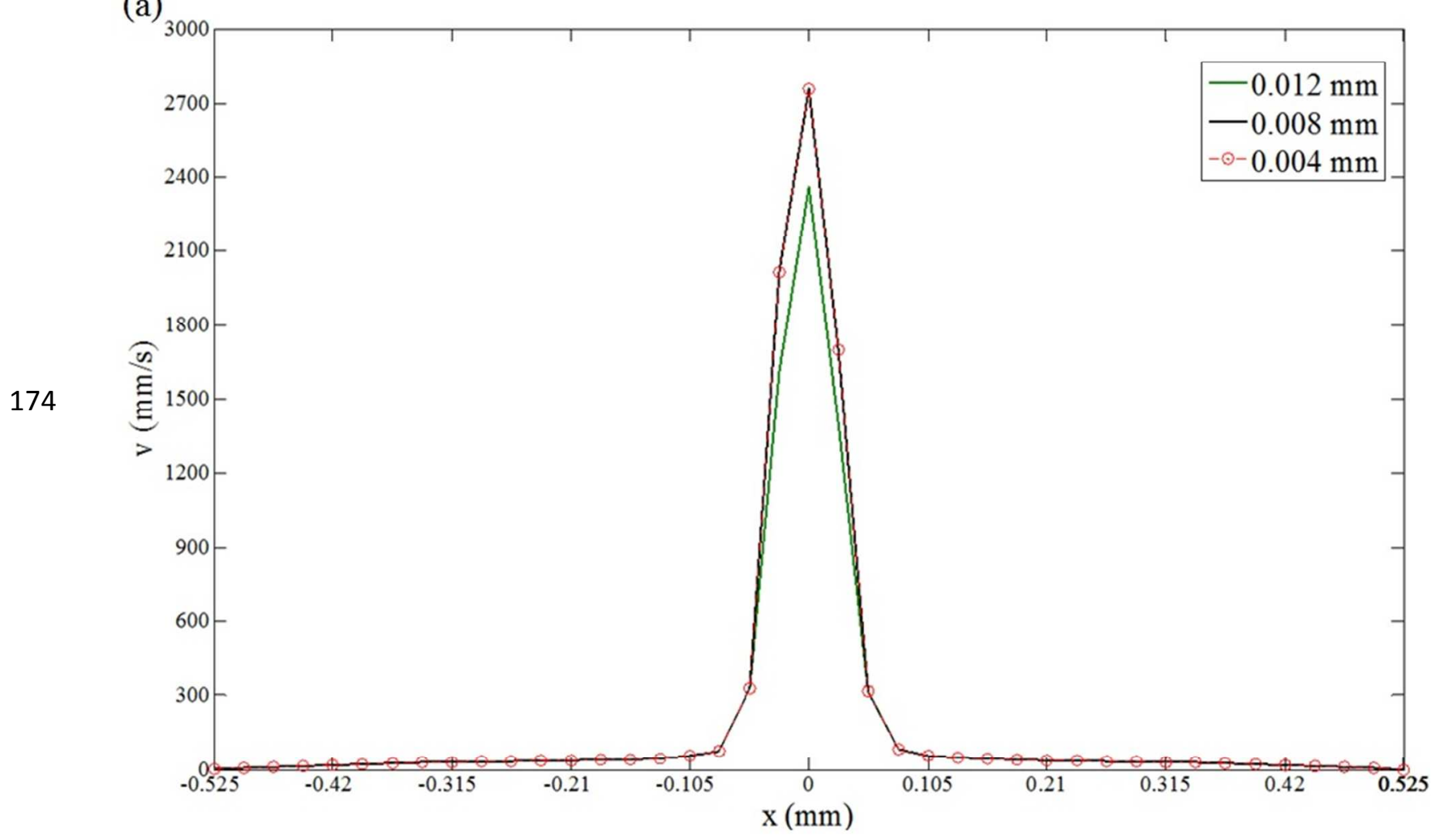

(b)

175

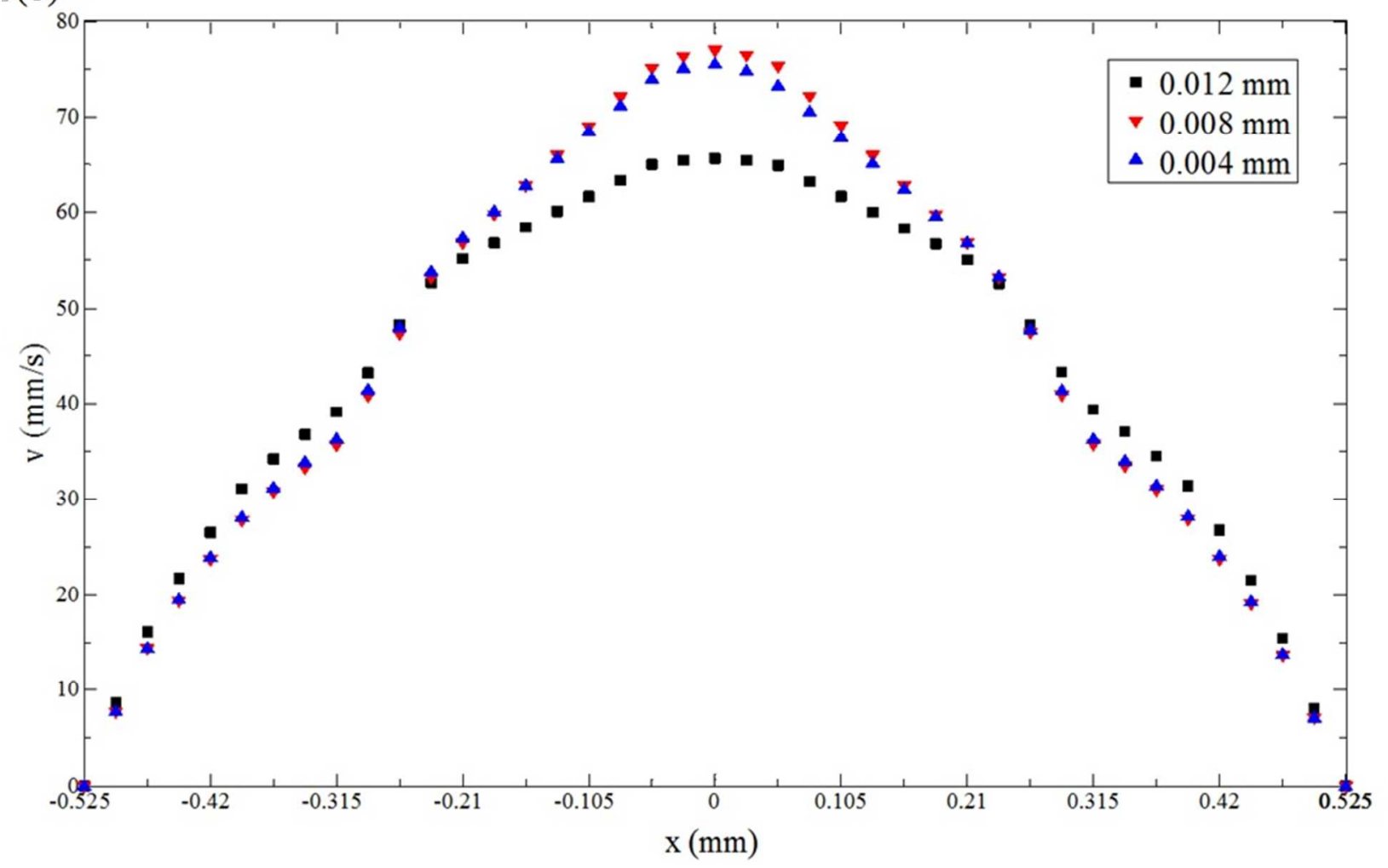


176

177

178

179

180

181

182

183

184

185

186

187

188

189

190

191

192

193

194

195

196

197

Fig.3 Validation of the numerical model of Newtonian/shear-thinning multiphase microfluidic system by comparison of axial velocity profile along transversal direction ( $x$ direction) at (a) $z=0.02 \mathrm{~mm}$ and (b) $\mathrm{z}=5.27 \mathrm{~mm}$ using different meshing grid sizes.

\section{Experimental section}

The Newtonian/shear-thinning two-phase co-flow is generated using a glass microcapillary device reported earlier. ${ }^{23} \mathrm{~A}$ glass slide was used as a substrate to support the capillary device, which is composed of an inner round capillary (World Precision Instruments, Inc) and an outer square capillary (Atlantic International Technology, Inc.).The round capillary has inner and outer diameters of $630 \mu \mathrm{m}$ and $1.0 \mathrm{~mm}$, and its tip was tapered to the desired diameter by a micropipette puller (Sutter Instrument, Inc.) to obtain an orifice with an inner diameter of about $49 \mu \mathrm{m}$. The tapered round capillary was fitted into the square capillary, which has an inner dimension of $1.05 \mathrm{~mm}$.Sincethe outer diameter of the round capillary matches with the inner dimension of the square capillary, coaxial alignment of the two capillaries is ensured. The dispersed phase was injected into the device through the circular capillary, while the continuous phase was injected in the same direction through the square capillary. The fluids were injected into the device through a flexible plastic tubing (Scientific Commodities Inc.), which was connected to syringe pumps (Longerpump, LSP01-2A) at controlled flow rates. Unless otherwise specified, the chemicals used in the study were supplied by Aladdin Reagents (Shanghai) Co., Ltd. In the present study, the dispersed phase was silicon oil, while the continuous phase was $2 \%$ (w/v) aqueous solution of sodium carboxymethyl cellulose (CMC) $(\mathrm{Mw}=250,000 \mathrm{Da}, \mathrm{DS}=1.2)$, which is a pseudo-plastic fluid and demonstrates shear-thinning behaviors. The rheological behavior of CMC solution can be characterized by two critical concentrations: ${ }^{27}$ a first critical 
198

199

200

201

202

203

204

205

206

207

208

209

210

211

212

213

214

215

216

217

218

219

220

CMC concentration indicates the transition to the semidilute network solution, while a second critical CMC concentration indicates the transition to the concentrated solution. Below the second critical concentration, the CMC solution becomes highly viscous and the dynamics is dominated by viscous effects; above the second critical concentration, the dynamics become dominated by elastic effects. For CMC solution with a nominal molecular weight of 700,000 Da and a degree of substitution of $0.65-0.85$, the first critical concentration is $\sim 1 \%$ while its second critical concentration is $2.5 \% .{ }^{27}$ The effect of concentration and molecular weight on the rheological behavior of aqueous CMC solutions has been investigated experimentally. ${ }^{28}$ Based on their measurements of the rheological properties, solutions of CMC with molecular weights of 90,000 Da, 250,000 Da, and 700,000 Da all exhibit predominantly shear-thinning behaviors over a shear rate of $0-1000 / \mathrm{s}$ in absence of yield stress as their concentration changes from $0.1 \%$ to $3.0 \%$. The average shear rate of CMC solution is below $1000 / \mathrm{s}$ by controlling flow rate in our work. Therefore, it is reasonable to assume that our $\mathrm{CMC}$ solution ( $\mathrm{Mw}=250,000 \mathrm{Da}, \mathrm{DS}=1.2)$ still has a predominantly viscous behavior; and the elastic effect can be ignored. ${ }^{28}$

We also prepared $5 \%(\mathrm{w} / \mathrm{v})$ aqueous solution of polyacrylamide (PAA, supplied by Wing Hing Chemical Co., Ltd) as the continuous phase to investigate the elastic effect on the breakup dynamics and droplet formation. The flow behavior inside the microcapillary device was monitored with an inverted microscope (Motic, ocular: WF10 $\times 18 \mathrm{~mm}$, object lens: EA4). A highspeed camera (Phantomv9.1 high speed camera) was connected to the microscope and the flow through the capillary was captured. The dripping-to-jetting transition using non-Newtonian systems was characterized experimentally, and the breakup dynamics in a non-Newtonian fluid system was compared with a Newtonian fluid system at the same Weber number and Capillary number using the same microdevice. For the Newtonian two-phase flow, silicone oil was also 
221

222

223

224

225

226

227

228

229

230

231

232

used as the dispersed phase, while $17 \% \mathrm{w} / \mathrm{v}$ aqueous solution of polyethylene glycol (PEG) $(\mathrm{Mw}=8,000 \mathrm{Da})$ was used as the continuous phase. The interfacial tension was measured by a Krussspinning drop tensiometer-SITE100. The respective viscosity of each solution was measured at different shear rates by a Brookfield DV-II+Pro programmable viscometer. Shearthinning behavior of the CMC solution was observed experimentally. The rheology data of CMC solution at all concentrations can be well represented by the well-known Cross model; ${ }^{27}$ therefore Cross model was used in curve-fitting of the experimental data, and the measured Cross model parameters (the zero shear viscosity $\eta_{0}=253.5 \mathrm{cP}$, infinite shear viscosity $\eta_{\infty}=60 \mathrm{cP}$, and the flow index $n=0.9$, as shown in Fig.4) were used in the simulation for computing the simulation results, which were subsequently compared with the experimental observations. Very small variations of viscosity were observed at different shear rates (see Fig.4), indicating that 17\% w/v PEG solution exhibits largely Newtonian properties. The concentration of PEG solution can affect the rheological property. For example, 7\% w/w PEG solution shows significantly different viscosity values at different shear rates, demonstrating a non-Newtonian behavior. ${ }^{29} 5 \% \mathrm{w} / \mathrm{v}$ PAA solution possesses shear-thinning behavior, as shown in Fig.4. As a typical viscoelastic liquid, the rheology property of PAA has been well investigated, and the storage modulus G' and loss modulus G" have been measured using the oscillatory shear rheometer. ${ }^{30}$ Based on the reported data, we find the relaxation time $\lambda=0.017 \mathrm{~s}$, according to Maxwell model of viscoelastic liquid. ${ }^{31}$ The relevant dimensionless numbers of viscoelastic liquid include Weissenberg number $W i$ and Elasticity number $E l$. The Weissenberg number is defined as the product of the relaxation time and a characteristicrate of deformation of the flow, and quantifies the nonlinear response of the liquid.

$$
W i=\frac{\lambda U}{D_{t}}=\frac{\lambda Q_{C P}}{D_{t}^{3}}
$$


244

245

246

247

248

249

250

251

252

253

254

255

256

where $U$ is the average axial flow velocity in the capillary device and $D_{t}$ refers to the dimension of the capillary tube. $W i$ is dependent on flow rate and varies in the range between 0.0041 and 0.041 in our experiments. The ratio between elastic and inertial effects is represented by the Elasticity number,

$$
E l=\frac{W i}{\operatorname{Re}}=\frac{\lambda \eta_{C P, 0}}{\rho_{C P} D_{t}^{2}}
$$

where Re refers to Reynolds number.El is thus independent on the flow rate based on the definition. In our experiments $E l=382$, indicating the dominating role of elastic effect relative to inertia effect. The physical properties of the fluids used in the investigation are shown in Table 1.

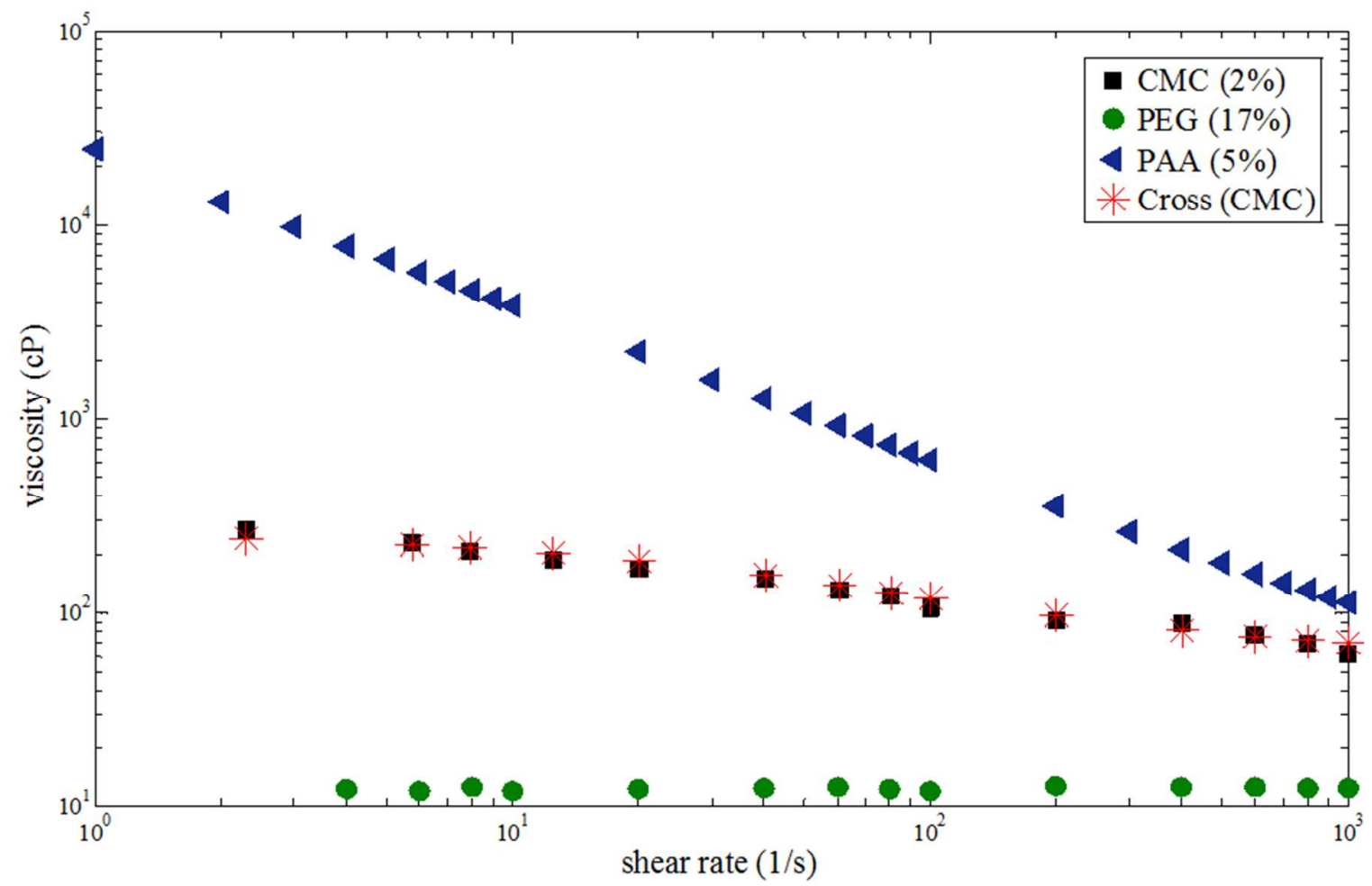

Fig.4 Rheological properties of the three aqueous polymer solutions: $2 \% \mathrm{w} / \mathrm{v}$ sodium carboxymethyl cellulose (CMC) in water (black squares), 17\% w/v polyethylene glycol (PEG) in water (green circles), 5\% w/v polyacrylamide (PAA) in water (blue triangle) and Cross model of CMC solution(red cross). 


\section{Table 1}

Physical property of the test fluids

\begin{tabular}{|c|c|c|c|c|c|}
\hline Test fluids & $\eta(\mathrm{cP})$ & $\rho(\mathrm{g} / \mathrm{ml})$ & $\begin{array}{l}\sigma(\mathrm{mN} / \mathrm{m}) \\
\text { (silicon oil } \\
\text { and } \mathrm{CMC} \text { ) }\end{array}$ & $\begin{array}{c}\sigma(\mathrm{mN} / \mathrm{m}) \\
\text { (silicon oil and } \\
\text { PEG) }\end{array}$ & $\begin{array}{c}\sigma(\mathrm{mN} / \mathrm{m}) \\
\text { (silicon oil and } \\
\text { PAA) }\end{array}$ \\
\hline Silicon oil & 10 & 0.963 & \multirow{4}{*}{7.12} & \multirow{4}{*}{9.624} & \multirow{4}{*}{10.176} \\
\hline $\mathrm{CMC}(2 \%)$ & $\begin{array}{c}253.5 \text { (zero- } \\
\text { shear rate) }\end{array}$ & 0.990 & & & \\
\hline PEG (17\%) & 13.6 & 1.053 & & & \\
\hline PAA (5\%) & $\begin{array}{c}24569 \text { (zero- } \\
\text { shear rate) }\end{array}$ & 0.992 & & & \\
\hline
\end{tabular}

\section{Results and discussion}

The breakup dynamics and dripping-to-jetting transition in Newtonian/shear-thinning multiphase microsystem are presented and discussed first, followed by discussion of elastic effect in droplet shape at the end of this section. Simulation and experiments of Newtonian/shear-thinning multiphase flow in the capillary microdevice have been conducted under the same flow conditions to observe the process of jet deformation and droplet formation. Silicon oil and CMC solution are used as dispersed phase and continuous phase, respectively. The flow pattern at successive time points from simulation is shown in Fig.5. The flow rates of the dispersed and continuous phasesare 5 and $7 \mathrm{ml} / \mathrm{h}$, respectively, with $C a_{\text {out }}=0.0631$ and $W e_{\text {in }}=5.147$. The transition regime is observed and the jet size changes along the length of jet. The dispersed phase

271 is purged out of the orifice, as shown by the inset in Fig.5a.The droplet grows in size and moves 272 downstream while it is still connected to the fluid neck through the orifice via a filament, as 273 shown in Fig.5b. The filament gradually becomes thinner (see Fig.5c) and finally breaks up intoa 
274 droplet (see Fig.5d). The process is repeated afterwards. The interface between the two phases in 275 Fig.5d is tracked by plotting the radius of interface along transversal direction ( $y$ direction) 276 versus the streamwise location along $z$ direction. The simulation result is represented by the solid 277 line, and is compared with experimental measurements indicated by the symbols. A reasonable 278 agreement, as shown in Fig.5e, validates the accuracy of the numerical model.

279 A satellite droplet is formed during the breakup of an elongated filament between two adjacent 280 droplets. The profile near the breakup point is highly asymmetric, with the droplet interface 281 being very steep towards the droplet while lying flat towards the neck of the jet. The existence of 282 satellite drops is intimately related to the non-linear properties of the fluid motion close to the 283 breakup point. ${ }^{32}$ When the filament breaks at both ends before merging with one of the parent 284 droplets, the filament separates from both neighboring parent droplets and recoils into a satellite 285 droplet. ${ }^{33}$ The number of satellite droplets and their relative sizes strongly depend on the viscosity 286 ratio of the dispersed phase to that of the continuous phase, and are also influenced by the initial 287 disturbance wavenumber, which is defined as $2 \pi a / \varepsilon$, where $a$ is the radius of filament and $\varepsilon$ is the 288 wavelength. ${ }^{34}$ At a small viscosity ratio, the slender center droplet undulates and pinches off at a 289 number of locations, generating a string of small satellite droplets. By contrast, when the 290 viscosity ratio is large, the internal flow leading to breakup is attenuated, resulting in the 291 formation of fewer satellite droplets. As the wave number increases, the ratio of radius of 292 satellite droplets in different birth regions relative to radius of parent droplet also increases. ${ }^{34}$ 

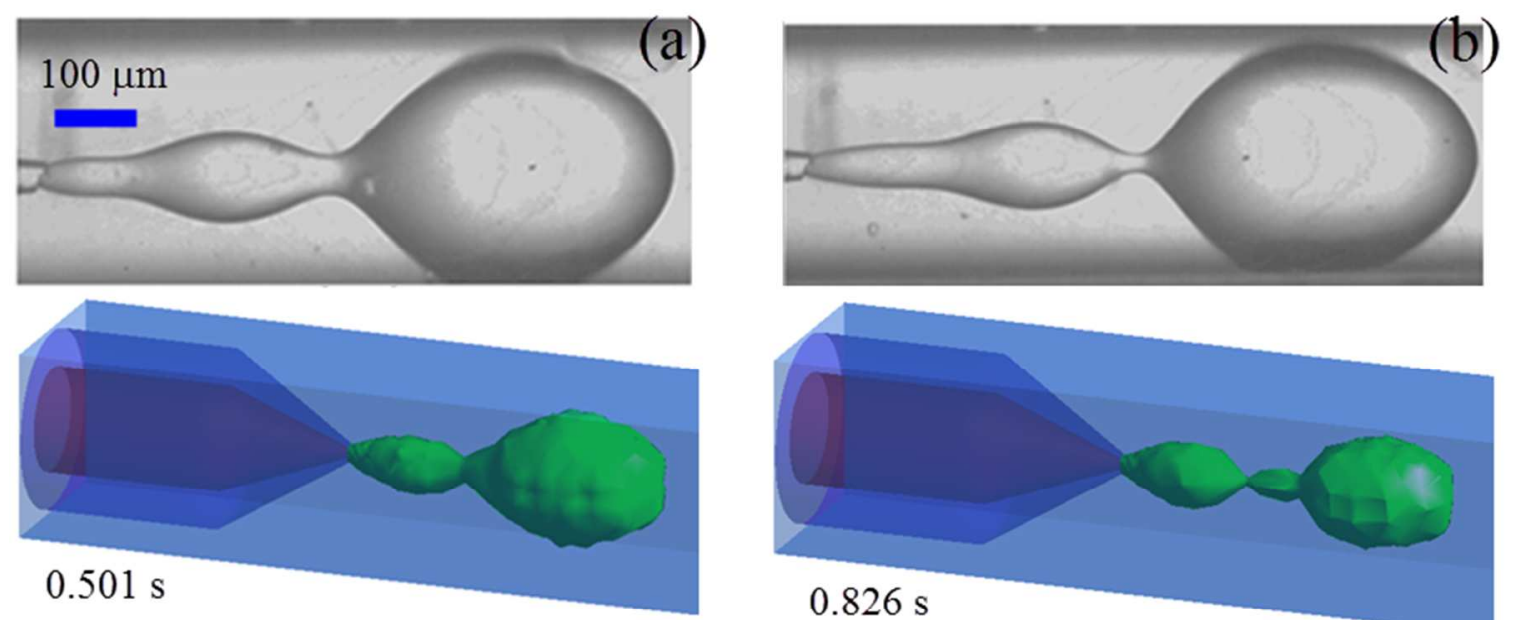

(b)
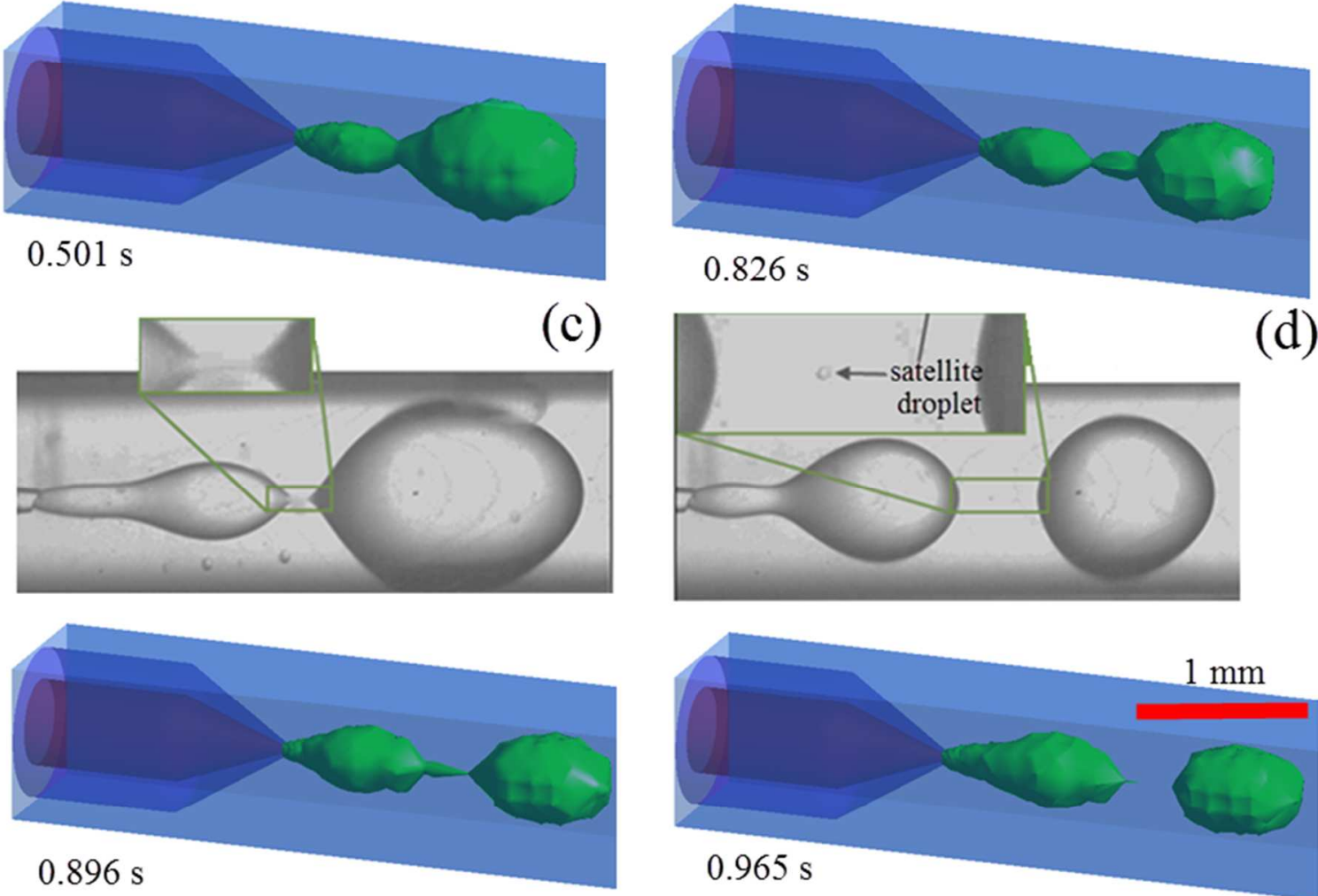

(d)
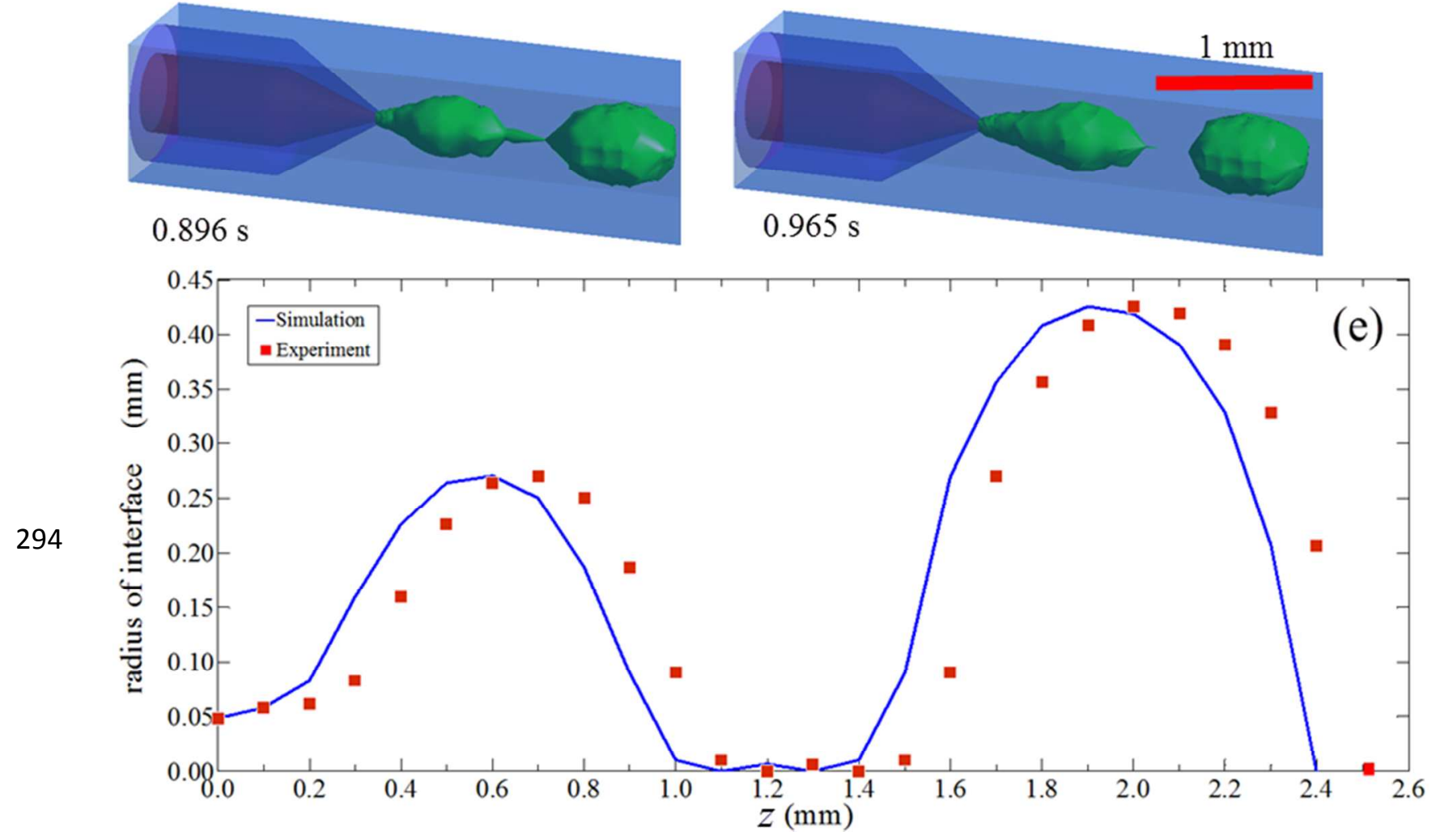

Fig.5 Time-lapse images of jet deformation and droplet formation from simulation (upper one in each sub-figure) and experiments (lower one in each sub-figure) using a Newtonian/shear- 
297

298

299

300

301

302

303

304

305

306

307

308

309

310

311

312

313

314

315

316

317

318

319

thinning two-phase co-flow system (silicon oil as dispersed phase and CMC solution as continuous phase). The blue and red scale bars are applicable for the experimental and simulation results respectively. (a) The dispersed phase is purged out of the orifice; (b) The main droplet is connected with the fluid neck via a thin filament; (c) The filament becomes even much thinner; (d) Satellite dropletoccurs after breakup of the jet; (e) Tracking of interface profile by both simulation and experimental observation.

Since the satellite droplets have a much smaller size than the main droplet, the global polydispersity of the resultant emulsion increases. To predict the occurrence of satellite droplets, the flow conditions that result in the formation ofsatellite droplets have been delineated using a state diagram, which shows the two-phase flow pattern as a function of Weber number and Capillary number.Three representative flow regimes are observed when the flow rate ratio is varied, as shown in Fig.6a: dripping, where droplets are formed in the vicinity of the capillary tip; intermediate, where the growing droplets move downstream while remaining connected to the fluid in the tip through a fluid neck; ${ }^{18}$ and jetting, where droplets breaks up from an extended jet with an incomplete retraction of the fluid neck. For the intermediate regime, the fluid neck retracts completely back to the tip after the droplet pinches off at the detachment point of the fluid neck. This regime is thus still regarded as dripping. ${ }^{18}$ Upon further increase of viscous or inertia force, for instance, by changing the flow rate of continuous or dispersed phase, the intermediate regime will transition to the jetting regime. Comparison of results from simulation and experiments shows a good agreement, confirming the validity of our model in capturing the physical behavior of the two-phase flow when a non-Newtonian fluid is used. As $W e_{i n}$ increases,the viscosity of the shear-thinning continuous phase decreases, when subjected to high shear rate. As a result, a lower viscousstress is exerted on the dispersed phase; the liquid thread is 
therefore not pulled further downstream. Consequently, the flow remains in the intermediate

321 regime rather than in the jetting regime. However, when $W e_{i n}$ is larger than 10 , jetting occurs

322 because of the larger inertial force. Based on experimental observation, satellite droplets appear

323 only in the intermediate regime, which is characterized as a dripping-to-jetting transition, ${ }^{18}$ as

324 shown in the state diagram in Fig.6b. Inertia is necessary to induce the formation of a satellite

325 droplet, because the droplet formation will be suppressed when the viscous force is dominant

326 over inertia. However, a very large inertial force will lead to formation of jetting and the liquid

327 thread will breakup into droplets further downstream. Consequently, the pinch-off time increases,

328 delaying the formation of the parent droplet and satellite droplets. This state diagram can help

329 predict the formation of undesirable satellite droplets in a Newtonian/shear-thinning two-phase

330 system, and can suggest operating conditions for eliminating satellite droplets. This ultimately

331 inspires production of monodisperse droplets by manipulating the breakup profile and

332 controlling the merging of satellite droplets with main droplets; yet these investigations are

333 beyond the scope of the present study. 
(a)Dripping $\left(\mathrm{Ca}_{\text {out }}=0.045, \mathrm{We}_{\text {in }}=0.052\right)$
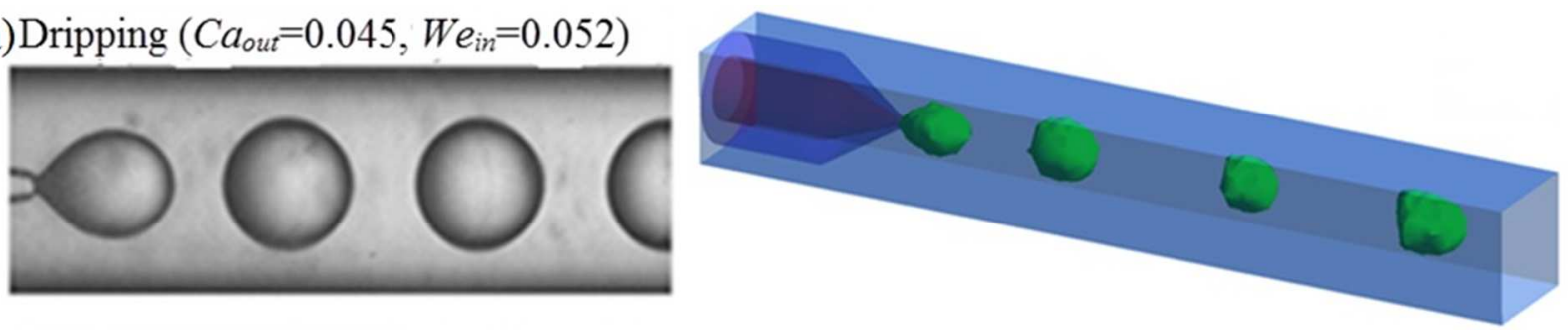

Intermediate $\left(C a_{o u t}=0.091, W e_{\text {in }}=5.15\right)$
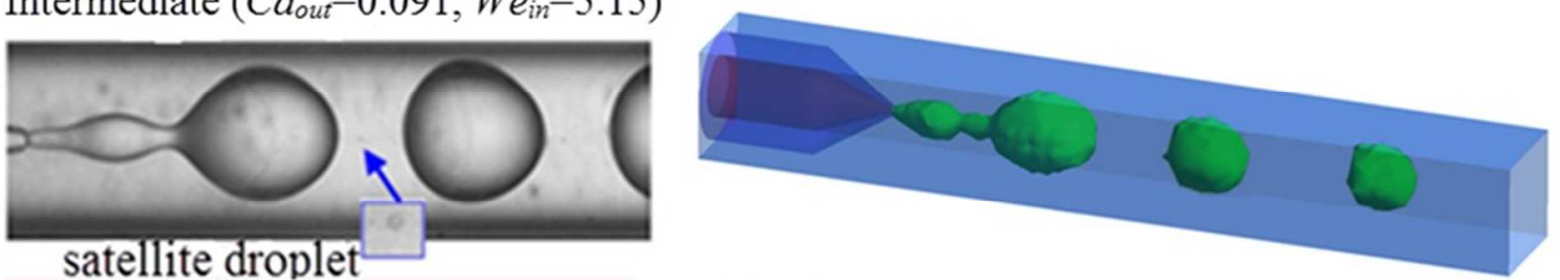

Jetting $\left(C a_{\text {out }}=0.18, W e_{\text {in }}=82.29\right)$
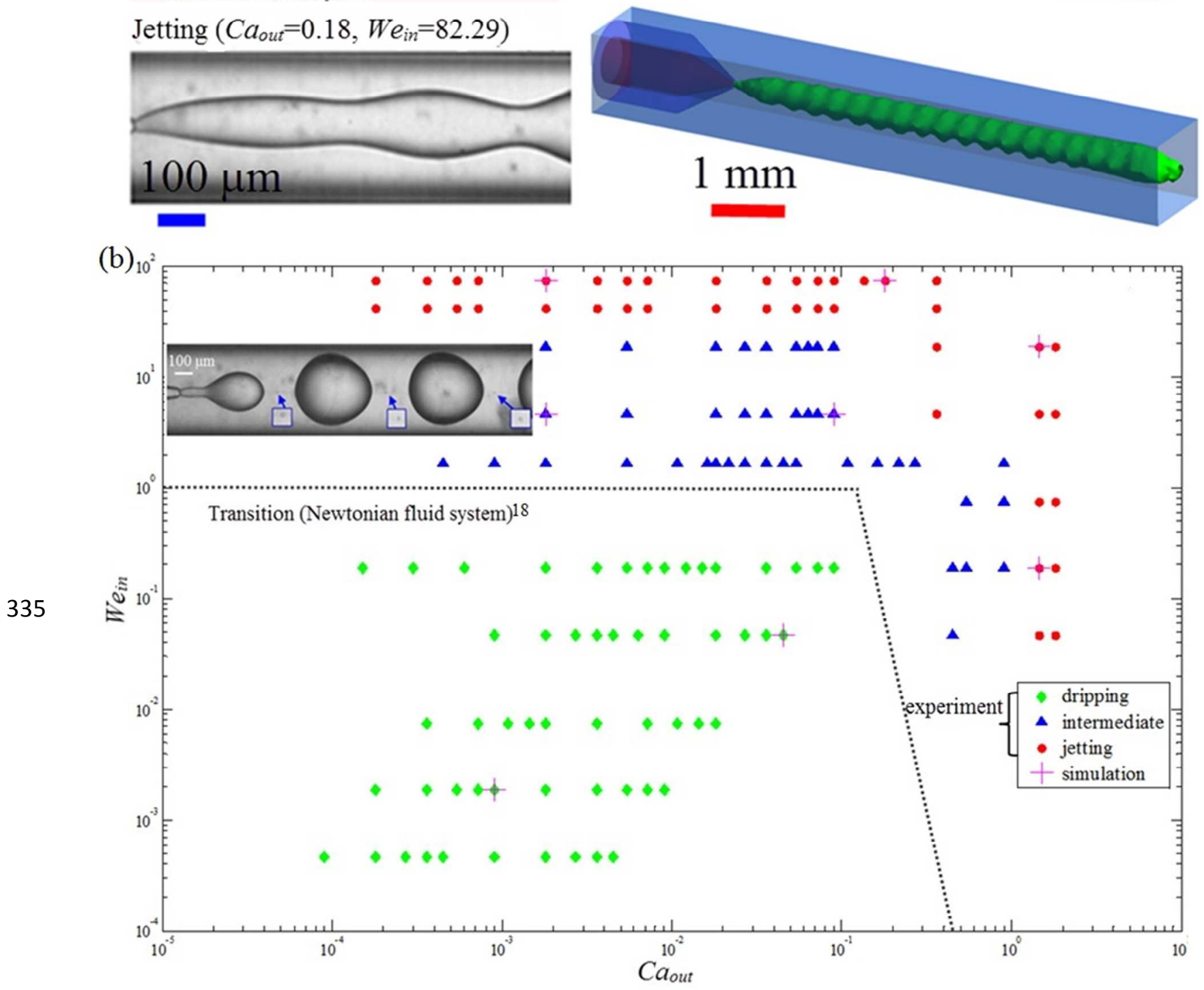
Fig.6(a) Comparison of results from simulation and experiments at different regimes using a Newtonain/shear-thinning two-phase coflow system (silicon oil as dispersed phase and CMC solution as continuous phase).The blue and red scale bars are applicable for the experimental and simulation results respectively. (b) State diagram showing the dripping-jetting transition as a function of $C a_{\text {out }}$ and $W e_{\text {in }}$. The inset shows the satellite droplets appearing periodically between adjacent parent droplets. The dash line (reproduced from reference. ${ }^{18}$ ) shows the dripping-tojetting transition in a purely Newtonian two-phase coflowsystem (deionized water and polydimethylsiloxane (PDMS) oils).

The breakup time is one of the determining factors on the droplet production rate. It is thus of fundamental interest and industrial relevance to investigate the breakup time when nonNewtonian fluids, for example, shear-thinning fluids, are used, in comparison to the counterpart of Newtonian multiphase system at the same $W e_{\text {in }}$ and $C a_{\text {out }}$. Droplet breakup occurs due to the interplay between the viscous stress and the interfacial tension between the two fluids. The viscous stress is closely associated with the shear rate, which varies greatly depending on the position and the shear rate, which affects the viscosity in all directions in the 3D flow. The highly deformed filament connecting the fluid neck with the droplet (see Fig.5b) is of particular interest, because it is the region where breakup occurs. We will therefore use the filament region, for instance, $z=0.87 \mathrm{~mm}$, as the focal point to characterize the shear-rate-dependent viscosity profile. Given the continuity of velocity at the interface and the small diameter of filament, the shear rate reaches a sharp peak at the interface between the two phases in the filament region. Due to shear-thinning characteristics of CMC solution, the viscosity will be dramatically reduced at the interface, as shown by the numerically calculated results of viscosity and shear rate as a function of the radial location along the transverse direction of the capillary device in Fig.7. The 
359

360

361

362

363

364

365

366

367 obtained at $r=0.375 \mathrm{~mm}$.

368

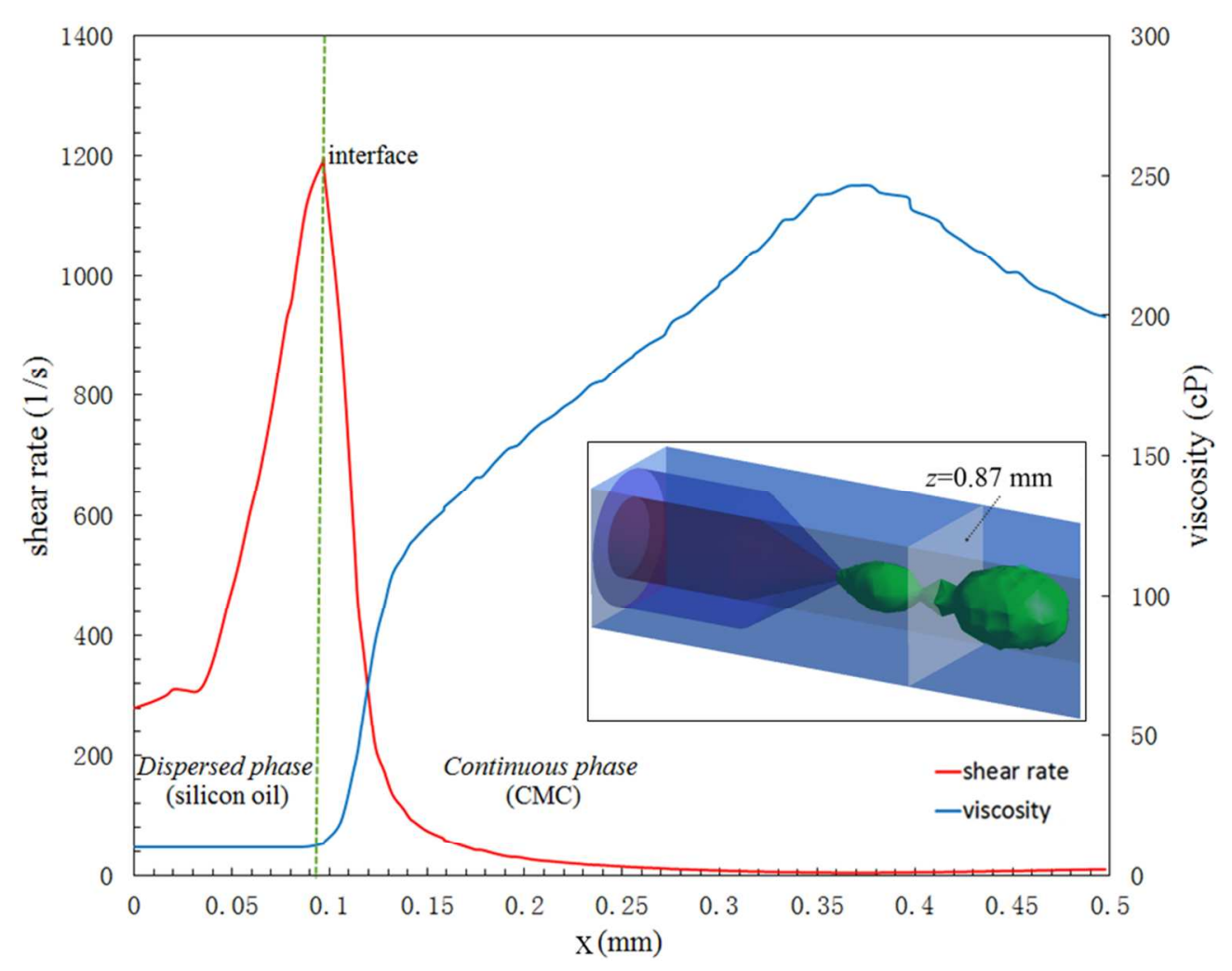

369

Fig. 7 The numerically calculated viscosity and shear rate as a function of the radial position

shear rate of the continuous phase in the co-flowing microfluidic device with a dimension of $D_{t}$ is related to the droplet diameter $D_{d}$ through, ${ }^{26}$

$$
\dot{\gamma} \sim \frac{Q_{C P}}{\pi D_{t}{ }^{3}\left[1-\left(\frac{D_{d}}{D_{t}}\right)^{2}\right]\left(1-\frac{D_{d}}{D_{t}}\right)}
$$

Given the flow condition in Fig.5, where $Q_{C P}=7 \mathrm{~mL} /$ hand $\frac{D_{d}}{D_{t}}=0.5, \dot{\gamma} \sim 1.65 / \mathrm{s}$. The calculated shear rate of the continuous phase is of the same order of magnitude as the simulation result. A local maximum shear rate of $9.68 / \mathrm{s}$ for continuous phase is obtained at the wall of collection tube, because of no-slip at thewall and large velocity gradient in boundary layer adjacent to the wall. The shear rate is reduced in the region far from the wall, and the minimum value of $3.87 / \mathrm{s}$ is

along the transverse direction of the capillary device of the Newtonian/shear-thinning two-phase 
371 coflow system (silicon oil as dispersed phase and CMC solution as continuous phase) at $z=0.87$

$372 \mathrm{~mm}$, which is illustrated in inset.

373 For shear thinning fluids, the viscosity is inversely proportional to the shear rate; thus the viscous

374 effect will be attenuated near regions with high shear rate, facilitating the pinchoff of the jet.

375 Consequently, the breakup process will be sped up so that a higher production rate of droplets

376 can be expected. To verify the hypothesis that shorter breakup time can be achieved when a

377 shear thinning fluid is involved, control tests have been conducted to monitor the breakup time

378 for the purely Newtonian two-phase system. The dimensionless pinching time,

$379 \hat{t}_{p}=t_{p} / t_{v}=t_{p} /\left(\eta_{0} d / \sigma\right)$, was measured experimentally at the same $W e_{\text {in }}$ and $C a_{\text {out }}$, where $t_{p}$ and

$380 t_{v}$ refer to the measured time interval between two successive pinching, and the viscous time

381 scale, respectively. When compared with the Newtonian system with constant viscosity, the

382 shear-thinning fluid near the liquid-liquid interface has a much reduced viscosity due to high

383 shear rate; as a result, at the same Capillary number, surface force dominates and the pinching is

384 faster when a shear-thinning fluid is used as the continuous phase. The dimensionless breakup

385 time is also reduced when $C a_{\text {out }}$ increases due to the enhanced viscous shear stress exerted on the

386 droplets, as shown in Fig. 8 where $W e_{i n}=1.852$. 


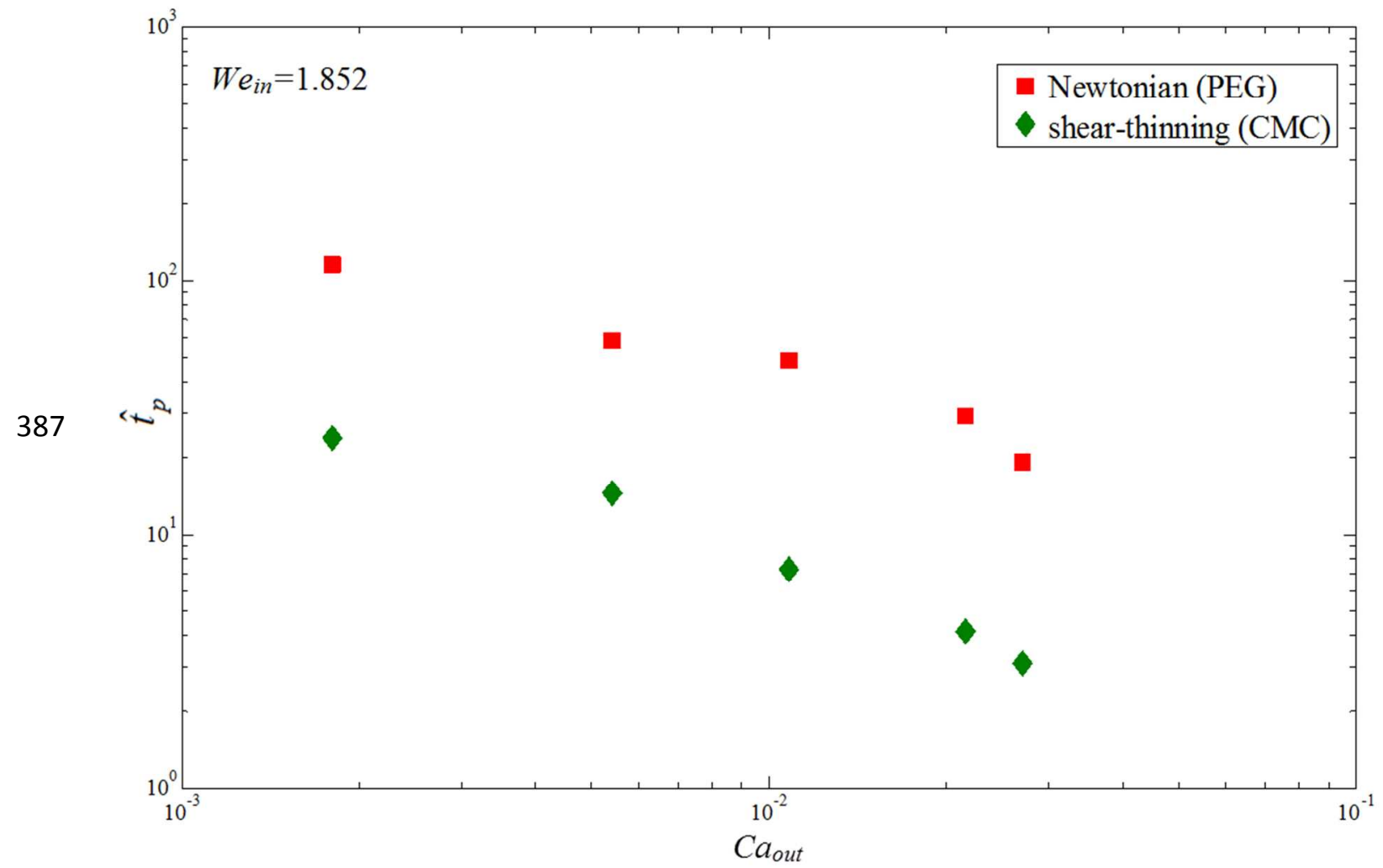

388

389

390

391

392

393

394

395

396

397

398

Fig.8 Comparison of the experimentally observed breakup time of jet into droplets using the Newtonian/shear-thinning two-phase coflow system (silicon oil as dispersed phase and CMC solution as continuous phase) versus Newtonian/Newtonian two-phase coflowsystem (silicon oil as dispersed phase and PEG solution as continuous phase) when $W e_{i n}=1.852$.

In the breakup process of liquid jet of dispersed phase, the droplets are formed and surrounded by the continuous phase. The droplet size is influenced by the viscous force and the surface tension force. Due to the reduced viscosity of the continuous phase, the droplet size is larger when a shear-thinning fluid is used at the same $W e_{\text {in }}$ and $C a_{\text {out }}{ }^{35}$ This is confirmed by the observed snapshots of droplets after breakup at the same Weber number but at different Capillary numbers in two different multiphase systems, as shown in Fig.9. In both cases, silicone oil is used as the dispersed phase, while solutions of PEG and CMC are used as the Newtonian and 
non-Newtonian continuous phases respectively. At the same Weber number of 1.852 , both systems show a decrease in the droplet size when the Capillary number is increased from 0.0018 to 0.054 (see Fig.10), due to the higher shear stress and the enhanced hydrodynamic focusing

402 effect by the continuous phase.

Newtonian/Newtonian system
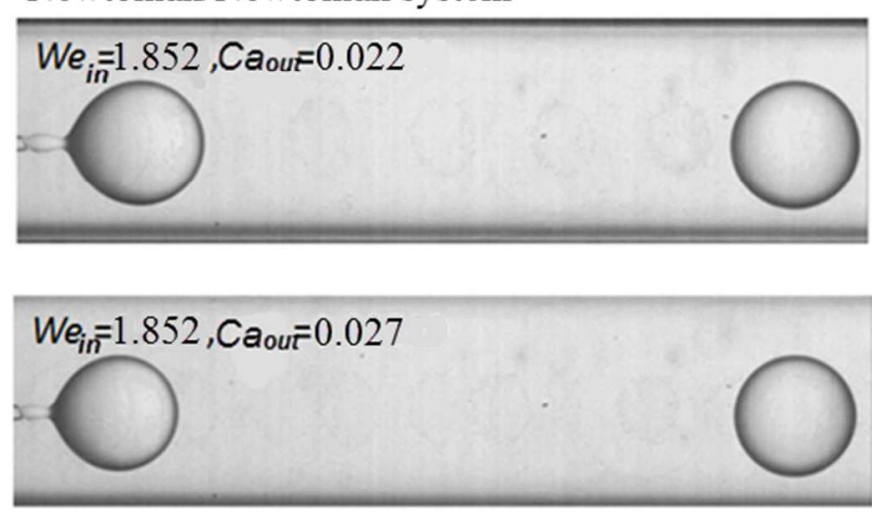

Newtonian/shear-thinning system $200 \mu \mathrm{m}$
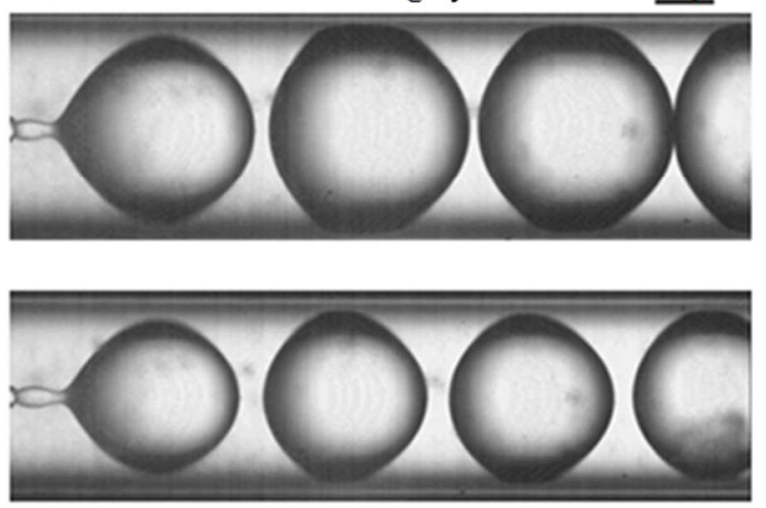

Fig.9 Comparison of droplet size betweenNewtonian/shear-thinning two-phase coflow system

405 (silicon oil as dispersed phase and CMC solution as continuous phase) and

406 Newtonian/Newtonian multiphase coflowsystem (silicon oil as dispersed phase and PEG solution

407 as continuous phase). 


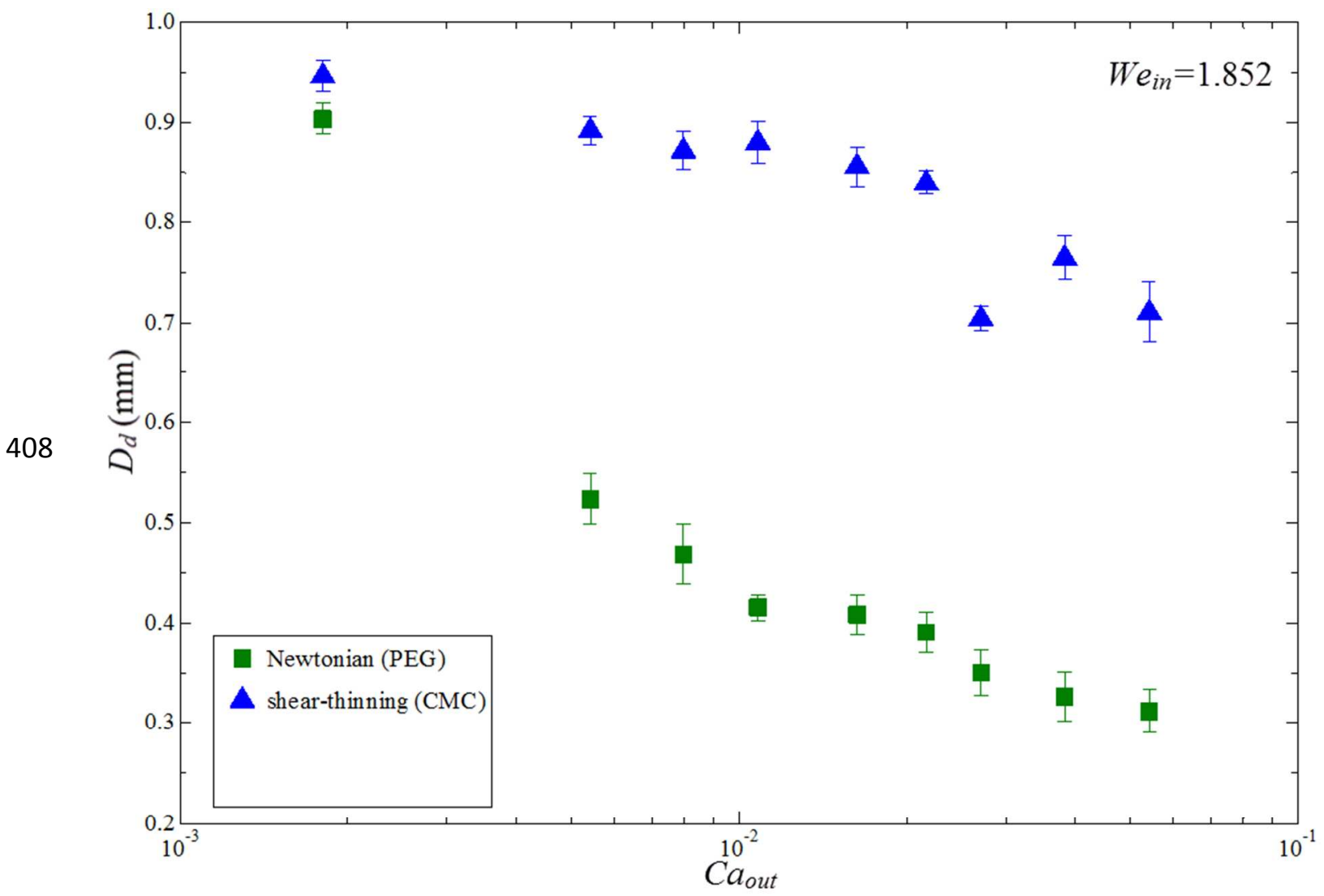

Fig.10 Comparison of the experimentally observed droplet diameter between the Newtonian/shear-thinning two-phase system (silicon oil as dispersed phase and CMC solution as continuous phase) and the Newtonian/Newtonian two-phase system (silicon oil as dispersed phase and PEG solution as continuous phase). $W e_{i n}=1.852$ in all cases.

413 Control of the droplet size is also an important aspect of droplet-based microfluidic applications.

414 For example, the volume of the droplet which contains reagents or analytes is one of the key 415 parameters that determinethe efficiency and the overall throughput of the system. ${ }^{36}$ Since non416 Newtonian fluids are ubiquitous in biochemical applications of microfluidics, it is also crucial to 417 control droplet size. To achieve smaller droplets, we vary the dimensions of the nozzles by 418 fitting a round capillary with radius $R$ into the square capillary (see Fig.11). At a constant flow 419 rate of outer-phase and constant Capillary number, evaluated based on the apparent viscosity, the 
shear rate increases with decreasing inner diameter of the collection capillary, leading to an increase in the shear stress and thus reduced droplet sizes, as shown by the observed droplets in

$$
C a_{\text {out }}=0.00181
$$

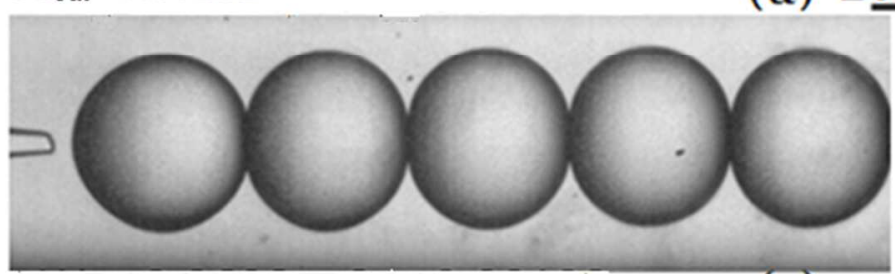

$C a_{\text {out }}=0.00363$

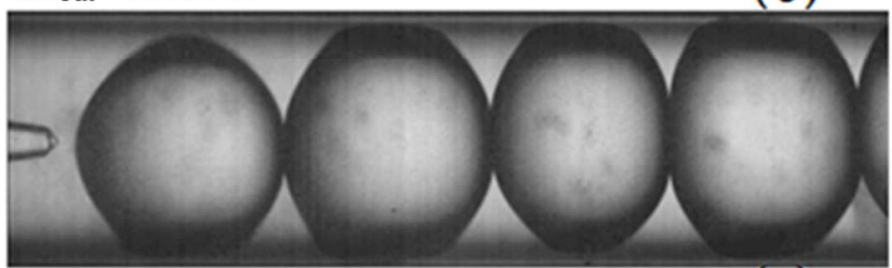

$$
C a_{\text {out }}=0.00903
$$

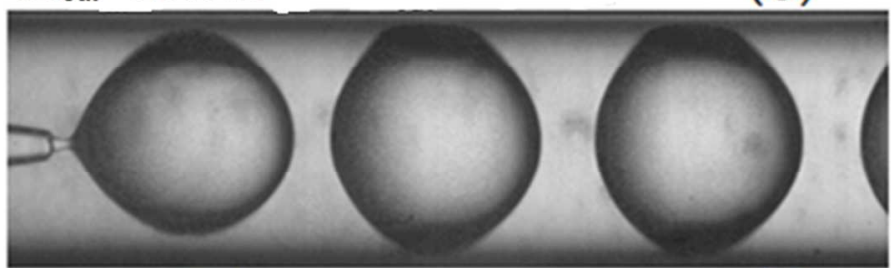

$$
C a_{\text {out }}=0.0181
$$

(g)

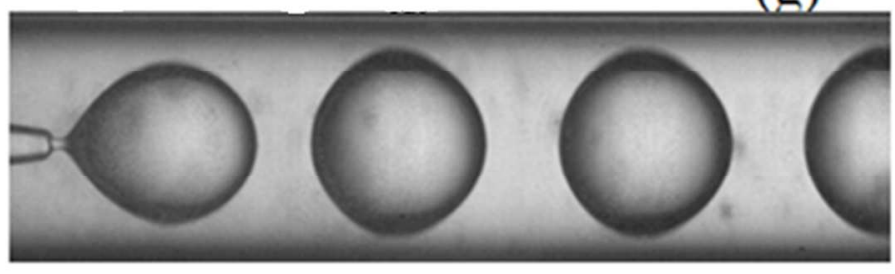

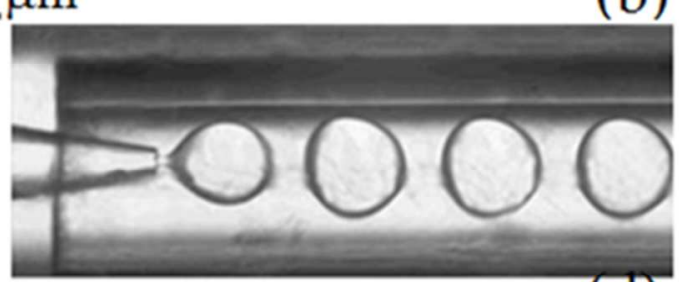
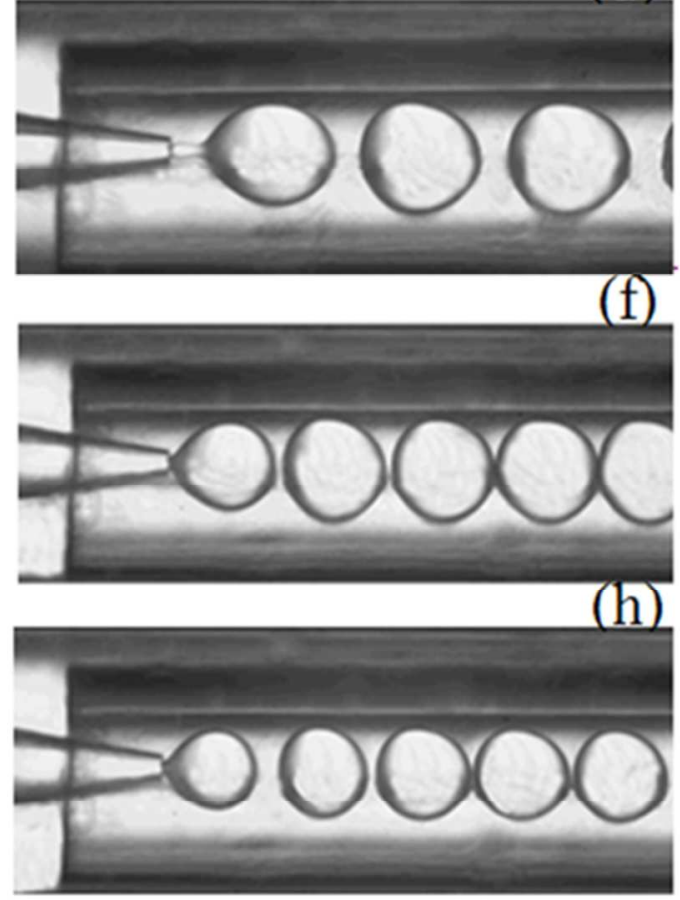

427 Fig.11 Observed droplets in collection tubes with different sizes $(R=0.5 \mathrm{mmin}$ a, c, e and g; 
429 dispersed phase and CMC solution as continuous phase). $C a_{\text {out }}=0.00181$ in a-b, $C a_{\text {out }}=0.00363$ in c-d, $C a_{\text {out }}=0.00903$ in e-f, and $C a_{\text {out }}=0.0181$ in $\mathrm{g}-\mathrm{h} . W e_{\text {in }}=0.206$ in all cases.

431

432

433

434

435

436

437

438

439

440

441

As $C a_{\text {out }}$ and the shear rate of continuous phase increase, the viscosity of continuous phase is reduced for shear-thinning fluids such as CMC; this results in larger droplets. ${ }^{35}$ However, when $C a_{\text {out }}$ increases beyond a critical value, the viscous drag can overcome the surface tension effects that would otherwise minimize the stretching of the fluid neck by drawing the fluid interfaces closer to the orifice. As a result, the fluid neck becomes stretched and elongated at high $C a_{\text {out }}$. The radius of fluid neck $r_{j}$ can be characterized by a scaling law that correlates the radius of fluid neck as a function of parameters including flow rate ratio, viscosity ratio and radius of collection tube, when the dispersed phase is not perturbed, ${ }^{37}$

$$
r_{j}=R\left[\frac{\left(1+\frac{Q_{D P} \eta_{D P}}{Q_{C P} \eta_{C P}}\right)^{0.5}-\left(1+\frac{Q_{D P}}{Q_{C P}}\right)}{\frac{\eta_{D P}}{\eta_{C P}}-2-\frac{Q_{D P}}{Q_{C P}}}\right]^{0.5}
$$

The shear rate is defined by,

$$
\dot{\gamma}=\frac{Q_{C P}}{\pi\left(R^{2}-r_{j}^{2}\right)\left(R-r_{j}\right)}
$$

442 443
The thinning of fluid neck occurs when it is stretched and elongated at high $C a_{\text {out }}$. Thus, the shear rate of the continuous phase (i.e., CMC in our case) starts to decrease, and the viscosity of continuous phase will increase for a shear-thinning fluid. As a result, the droplet size will start to be reduced beyond a critical $C a_{\text {out }}$, as shown in Fig. 12 when $W e_{i n}=0.206$. When the inner radius of the collection tube is $0.3 \mathrm{~mm}$, the droplet size increases from $0.40 \mathrm{~mm}$ to $0.55 \mathrm{~mm}$ as $C a_{\text {out }}$ is increased from $1.5 \times 10^{-4}$ to 0.0053 , while the droplet size is reduced to $0.38 \mathrm{~mm}$ when $C a_{\text {out }}$ is further increased to 0.015 . At a given Weber number, the droplet size first increases below a 
449 certain critical Capillary number due to the reduced viscosity, and then decreases above the 450 critical Capillary number, when the viscosity starts to increase again. The correlation between 451 the droplet size and the Capillary number in the Newtonian/shear-thinning two-phase system is 452 different from that in the Newtonian/Newtonian two-phase system, which normally shows that 453 droplet size scales inversely with the Capillary number of the continuous phase in a monotonous 454 fashion. ${ }^{38}$ The different correlation highlights the complex viscosity effect towards the control 455 over emulsion droplets generated with fluid-fluid systems involving shear-thinning non456 Newtonian fluids. This understanding can provide information needed for designing 457 microdevicesfor generating droplets with well-defined volumes when shear-thinning non458 Newtonian multiphase systems are involved. For example, given certain flow conditions and 459 fluid properties (thus $W e_{\text {in }}$ and $C a_{\text {out }}$ are known), an analysis can be made to determine the size of 460 collection tube of the microdevice, according to the expected size of droplets in demand. 


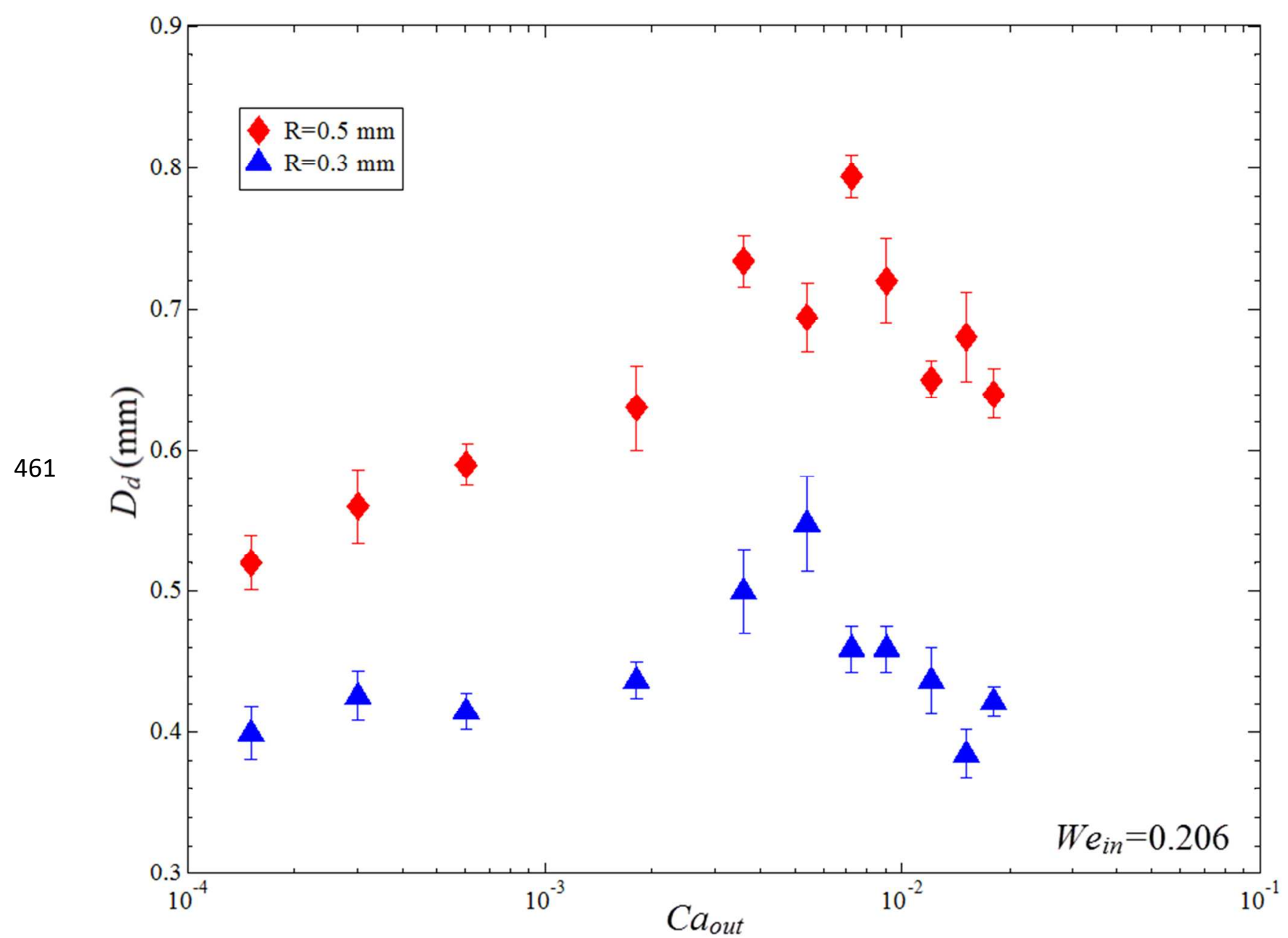

462 Fig.12 Experimental result of droplet size as a function of the Capillary number for a given 463 collection tube in a Newtonian/shear-thinning two-phase system (silicon oil as dispersed phase 464 and CMC solution as continuous phase). $W e_{i n}=0.206$.

465 When a Newtonian liquid is surrounded by a shear-thinning liquid with pronounced elastic 466 property, the droplet breakup dynamics gets modified. We observe flow patterns at different 467 ratios of flow rate of 5\% w/v PAA solution, which is the viscoelastic continuous phase, to that of 468 silicon oil as dispersed phase, as shown in Fig.13. The flow rate of dispersed phase is fixed at 1 $469 \mathrm{ml} / \mathrm{h}$ in Fig.13a-f, and $10 \mathrm{ml} / \mathrm{h}$ in Fig.13g-i. The elastic forces from viscoelastic continuous phase 470 can help to overcome interfacial tension, and thus facilitate transition to jetting at smaller 
471 magnitudes of the viscous forces. Compared to the aforementioned Newtonian/Newtonian or

472 Newtonian/shear-thinning multiphase microsystem where droplets normally adopt a spherical or

473 nearly spherical shape, the droplets experience significant deformation in the viscoelastic non-

474 Newtonian continuous phase. For instance, the droplets adopt an elliptical shape after breakup

475 and relax into pointed shapes. Droplets become more pointed as the radius decreases and the

476 flow rate ratio increases before it transitions to the jetting regime (see Fig.13a-f). The degree of

477 droplet deformation has been characterized based on Taylor's analysis, ${ }^{39}$

478

$$
D_{f}=\frac{L-W}{L+W}
$$

where $L$ is the half-length and $W$ the half-breadth of the droplet. $D_{f}$ increases with increasing

Weissenberg number (thus more pronounced elastic effect), leading to formation of droplets with more pointed shape, as shown in Fig.14. This observation is consistent with the previous finding dynamics and droplet formation in multiphase microfluidic system.

(a) $Q_{d} / Q_{d}=5, W i=0.020$

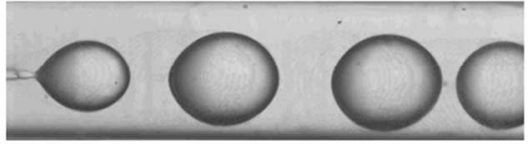

(d) $Q_{d} / Q_{d}=8, W i=0.033$

486 (b) $Q_{d} / Q_{d}=6, W i=0.024$

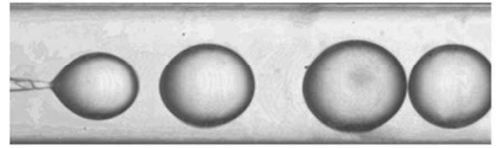

(e) $Q_{d} / Q_{d}=9, W i=0.037$

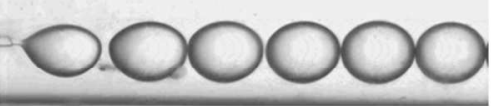

(h) $Q_{d} / Q_{d}=0.2, W i=0.0082$ (f) $Q_{d} / Q_{d}=10, W i=0.041$
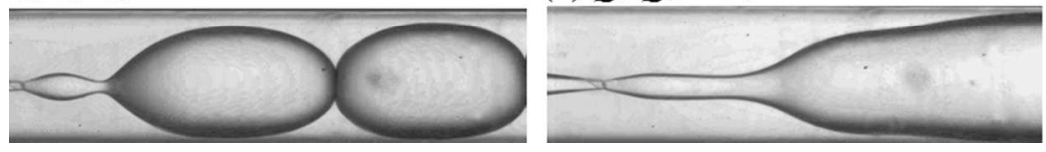

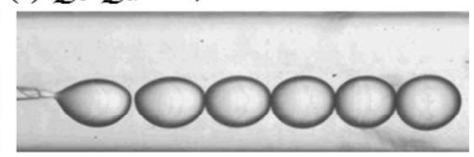

(i) $Q_{d} / Q_{d}=1, W i=0.041$

(c) $Q_{d} / Q_{d}=7, W i=0.029$
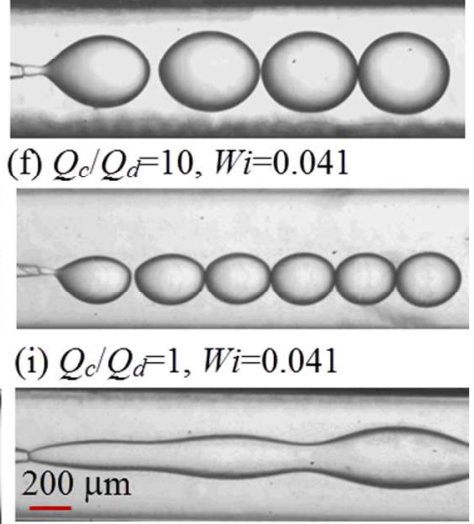
Fig.13 Flow patterns at selected flow rate ratios of continuous phase over dispersed phase in a 488 Newtonian/elastic-shear-thinning two-phase system (silicon oil as dispersed phase and PAA 489 solution as continuous phase). $Q_{d}=1 \mathrm{ml} / \mathrm{h}$ for (a)-(f), and $Q_{d}=10 \mathrm{ml} / \mathrm{h}$ for (g)-(i).

490

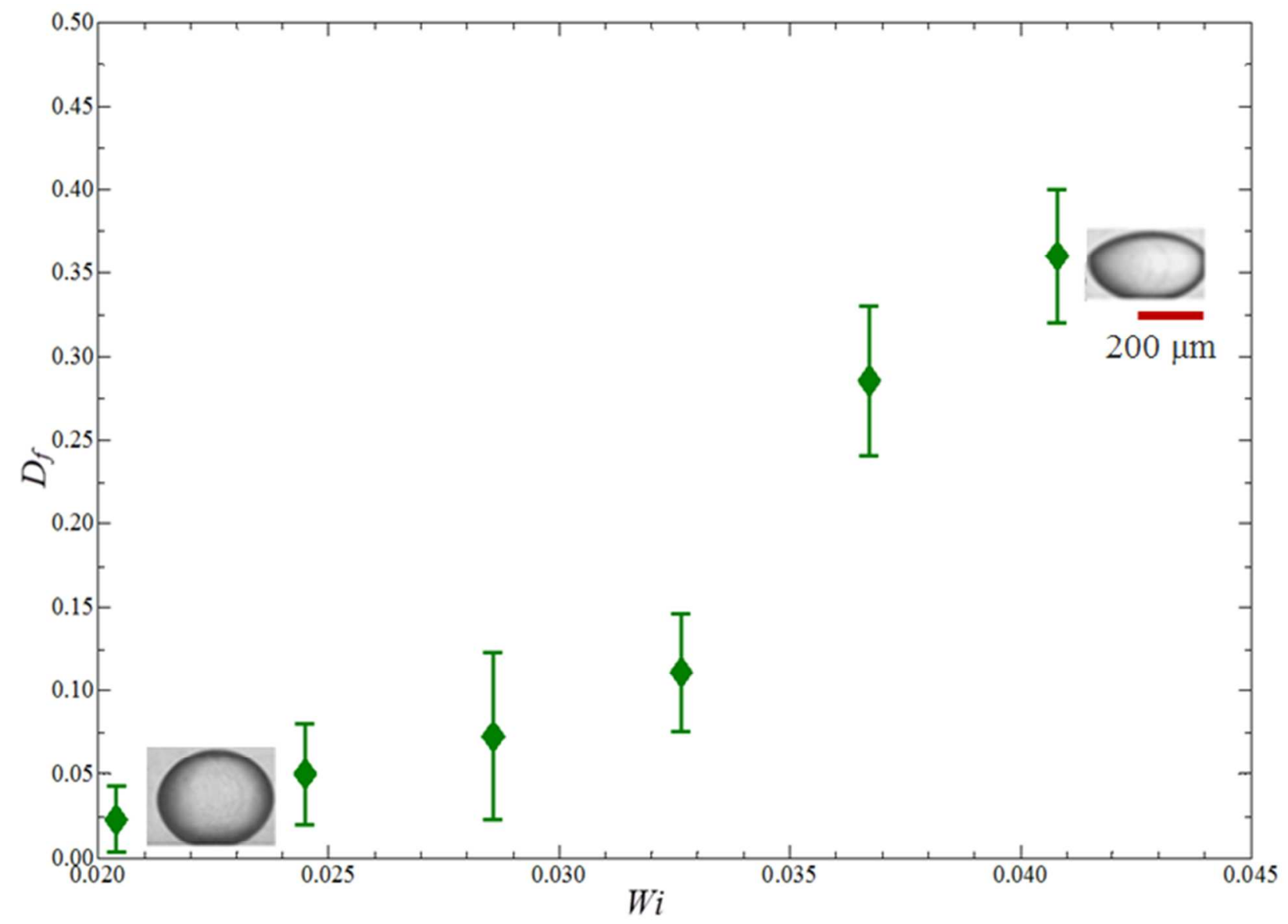

Fig.14 Degree of deformation of droplet as a function of the Weissenberg number in a

492 Newtonian/elastic-shear-thinning two-phase system (silicon oil as dispersed phase and PAA solution as continuous phase). The inset images show droplet adopts nearly spherical shape at low $W i$, while pointed shape at high $W i$, respectively. The scale bar applies for both inset images.

\section{Conclusions}


497 The work reports investigation of emulsion formation using a Newtonian/shear-thinning two498 phase microsystem. With a shear-thinning continuous phase, the droplet dynamics is 499 characterized to predict the dripping-to-jetting transitions under different flow conditions (as 500 function of Weber number and Capillary number), where undesirable satellite droplets may be 501 formed. Inertial effects have been shown to be important for inducing the formation of a satellite 502 droplet, while the droplet formation can be suppressed by increasing viscous effect. Due to the 503 shear-thinning characteristics of the continuous phase used in the present study, the viscosity is 504 dramatically reduced at the interface; viscous effect is therefore attenuated, leading to a faster 505 breakup and a larger droplet size. Emulsions generated with non-Newtonian fluids are routinely 506 involved in drug delivery and other biochemical applications. Accurate dosing must be ensured 507 for reliable operation, and this requires excellent control over the droplet size.We also identify 508 the correlation between the droplet size and Capillary number, potentially enabling a higher 509 degree of control over the size of emulsion droplet generated with shear-thinningfluids. Finally, a 510 viscoelastic fluid is used as continuous non-Newtonian phase, and the degree of Newtonian 511 droplet deformation increases with increasing Weissenberg number of suspending non512 Newtonian fluid, indicating the important impact of elasticity on the droplet shape. Our work 513 provides a first framework for understanding a range of behaviors in non-Newtonian multiphase 514 microsystems, although we recognize the vast variety of non-Newtonian fluids besides the two 515 types (shear-thinning solution with or without elastic property) we have investigated and the 516 large regions of unexplored operating conditions. For example, it is challenging to generate 517 droplets with non-Newtonian fluids in aqueous two phase systems (ATPS) due to much lower 518 interfacial tension when compared with oil-water systems. ${ }^{41-42}$ Furthermore, it is also of great 519 interest to investigate the cases when non-Newtonian fluid is used as the dispersed phase, rather 
520 than just the continuous phase. Improvement in the polydispersity of the resultant droplets can

521 also be achieved potentially by developing and implementing methods to dynamically control the

522 merging of satellite droplets with the main droplets. This approach of elimination of satellite

523 droplets will also lead to droplets with higher size uniformity in applications that inevitably

524 involve non-Newtonian multiphase flows.

\section{Acknowledgement}

526 This research was supported by the Early Career Scheme (HKU 707712P) and the General 527 Research Fund (HKU 719813E) from the Research Grants Council of Hong Kong, the Young 528 Scholar's Program (NSFC51206138/E0605) from the National Natural Science Foundation of 529 China, the Basic Research Program-General Program (JC201105190878A) from the Science and 530 Technology Innovation Commission of Shenzhen Municipality, as well as the Seed Funding 531 Programme for Basic Research (201211159090) and Small Project Funding (201109176165) 532 from the University of Hong Kong.

\section{Reference}

5341 H.C. Shum, D. Lee, I. Yoon, T. Kodger and D.A. Weitz, Langmuir, 2008, 24, 7651-7653.

5352 T. Kong, J. Wu, M.To, K.W.K. Yeung and H.C. Shum, Biomicrofluidics, 2012, 6, 034104.

5363 C.I. Zoldesi, P. Steegstra and A. Imhof, J. Colloid Interface Sci., 2007, 308, 121-129.

5374 J.K. Nunes, S.S.H. Tsai, J. Wan and H.A. Stone, J. Phys. D Appl. Phys., 2013, 46, 114002.

5385 C.H. Choi, H. Yi, S. Hwang, D.A. Weitz and C. Lee, Lab Chip, 2011, 11, 1477. 
539

540

541

542

543

544

545

546

547

548

549

550

551

552

553

554

555

556

557

558

559

560

561

6 C.M. Hwang, A. Khademhosseini, Y. Park, K. Sun and S. Lee, Langmuir, 2008, 24, 68456851.

7 J.Wu, T. Kong, K.W.K.Yeung, H.C. Shum, K.M.C. Cheung, L. Wang, and M.K.T. To, Acta Biomaterialia, 2013,9(7), 7410-7419.

8 Ö.E. Y1ldırım and O.A. Basaran, J. Non-Newtonian Fluid Mech., 2006, 136, 17-37.

9 A.R. Abate, M. Kutsovsky, S. Seiffert, M. Windbergs, L.F.V. Pinto, A.Rotem, A.S. Utada and D.A. Weitz, Adv. Mater., 2011, 23, 1757-1760.

10 C. L. A. Berli, J. Colloid Interface Sci., 2010, 349, 446-448.

11 T. Nguyen, Y. Xie, L. J. de Vreede, A. van den Berg and J.C.T. Eijkel, 16th Int. Conf. on Miniaturized Systems for Chemistry and Life Sciences, 2012, 1987-1989.

12 D.J. Mcclements, Crit. Rev. Food Sci.Nutr., 2007, 47, 611-649.

13 A. Budhian, S.J. Siegel and K.I. Winey, Int. J. Pharm., 2007, 336, 367-75.

14 C. Berkland, M. King, A. Cox, K. Kim and D. Pack, J. Control Release, 2002, 82, 137-47.

15 R.M. Erb, D. Obrist, P.W. Chen, J. Studer and A.R. Studart, Soft Matter, 2011, 7, 8757.

16 M.S.N. Oliveira and G.H. McKinley, Phys. Fluids,2005, 17,071704.

17 J. Eggers1 and E. Villermaux, Rep. Prog. Phys., 2008, 71,036601.

18 A.S. Utada, A. Fernandez-Nieves, H.A. Stone and D.A. Weitz, Phys. Rev. Lett., 2007, 99, 094502.

19 R.G.Larson, Thestructure and rheology of complex fluids, Oxford University Press, Oxford, 1999.

20 S.Yang, J.Y.Kim, S.J.Lee, S.S. Lee, and J.M.Kim, Lab Chip, 2011, 11, 266.

21 L.Derzsi, M.Kasprzyk, J.P.Plog, and P.Garstecki, Phys. Fluids, 2013,25, 092001.

22 G. Pangalos, J.M. Dealy and M.B. Lyne, J. Rheol.,1985, 29, 471-491. 
562

563

564

565

566

567

568

569

570

571 $572 \quad$ University, 2000.

573

574

575

576

577

578

579

580

581

582

583

584 Heinemann, 1988.

23 A. Sauret, C. Spandagos and H.C. Shum, Lab Chip, 2012, 12, 3380.

24 L.Sang, Y.Hong and F. Wang, Microfluid Nanofluid, 2009,6, 621-635.

25 C.L. Tucker, Hanser Publishers, New York, 1989.

26 Y. Hong and F. Wang, Microfluid Nanofluid, 2007, 3,341-346.

27 A. Benchabane and K. Bekkour, Colloid. Polym. Sci., 2008, 286 (10), 1173-1180.

28 K. Benyounes, 13th SGEM GeoConference on Science and Technologies In Geology, Exploration and Mining, 2013, 951-95.

29 S.D. Geschiere, I.Ziemecka, V. van Steijn, G.J.M. Koper, J.H. van Esch and M.T. Kreutzer, Biomicrofluidics, 2012,6, 022007.

30 A. Kreiba, The Rheological Properties of Aqueous Polyacrylamide Solutions, Concordia

31 R. G. Larson, Constitutive Equation for Polymer Melts and Solutions, Butterworth-

32 J. Eggers, ZAMM · Z. Angew.Math. Mech., 2005, 85,400-410.

33 M.Rohani, F.Jabbari and D. Dunn-Rankin, Phys. Fluids, 2010, 22, 107103.

34 M. Tjahjadi, H.A. Stone and J. M. Ottino, J. Fluid Mech.,1992, 243,297-317.

35 G.I. Taylor, Proc. R. Soc.LondonSer.A, 1932, 138, 41-47.

36 H. Song, D.L. Chen and R.F.Ismagilov, Angew. Chem. Int. Ed., 2006, 45, 7336-7356.

37 A. Sauret and H.C. Shum, Int.J.Nonlin. Sci. Num., 2012, 13, 351-362.

38 C.A. Stan, S.K.Y. Tang and G.M. Whitesides, Anal. Chem., 2009, 81, 2399-2402.

39 G.I. Taylor, Proc. Roy.Soc.A, 146 (1934) 501.

40 D.C. Tretheway and L.G. Leal, J. Non-Newtonian Fluid Mech., 2001, 99, 81-108.

41 Y.Song, A. Sauret and H.C. Shum, Biomicrofluidics, 2013,7, 061301. 
58542 A.Sauret and H.C. Shum, Appl. Phys. Lett.,2013, 100, 154106. 